\title{
YOUNG GALAXY CANDIDATES IN THE HUBBLE FRONTIER FIELDS. III. MACS J0717.5+3745
}

\author{
N. Laporte ${ }^{1}$, L. Infante ${ }^{1}$, P. Troncoso Iribarren ${ }^{1,2}$, W. Zheng ${ }^{3}$, A. Molino ${ }^{4,5}$, F. E. Bauer ${ }^{1,6,7}$, D. Bina ${ }^{8}$, \\ Tom Broadhurst ${ }^{9,10}$, I. Chilingarian ${ }^{11,12}$, X. Huang ${ }^{13}$, S. Garcia ${ }^{1,2}$, S. Kim ${ }^{1,2}$, R. Marques-Chaves ${ }^{14,15}$, J. Moustakas ${ }^{16}$, \\ R. Pelló ${ }^{8}$, I. Pérez-Fournon ${ }^{14,15}$, X. Shu ${ }^{17,18}$, A. Streblyanska ${ }^{14,15}$, and A. Zitrin ${ }^{19,20}$ \\ ${ }^{1}$ Instituto de Astrofísica and Centro de Astroingeniería, Facultad de Física, Pontificia Universidad Católica de Chile, \\ Vicuña Mackenna 4860, 7820436 Macul, Santiago, Chile \\ ${ }^{2}$ Centro de Astro-Ingeniera, Pontificia Universidad Catlica de Chile, Santiago, Chile \\ ${ }^{3}$ Department of Physics and Astronomy, Johns Hopkins University, Baltimore, MD 21218 \\ ${ }^{4}$ Instituto de Astronomía, Geofísica e Ciências Atmosféricas, Universidade de São Paulo, Cidade Universitária, 05508-090, São Paulo, Brazil \\ ${ }^{5}$ Instituto de Astrofísica de Andalucá-CSIC, Glorieta de la Astronomía, s/n. E-18008, Granada, Spain \\ ${ }^{6}$ Millennium Institute of Astrophysics, Vicuña Mackenna 4860, 7820436 Macul, Santiago, Chile \\ Space Science Institute, Boulder, CO 80301 \\ ${ }^{8}$ IRAP, CNRS-14 Avenue Edouard Belin-F-31400 Toulouse, France \\ ${ }^{9}$ Department of Theoretical Physics, University of Basque Country UPV/EHU, Bilbao, Spain \\ ${ }^{10}$ IKERBASQUE, Basque Foundation for Science, Bilbao, Spain \\ ${ }^{11}$ Smithsonian Astrophysical Observatory, 60 Garden St. MS09, Cambridge MA 02138, USA \\ ${ }^{12}$ Sternberg Astronomical Institute, Moscow State University, 13 Universitetsky prospect, Moscow, 119992, Russia \\ ${ }^{13}$ Department of Astronomy, University of Science and Technology of China, Hefei, Anhui 230026, China \\ ${ }^{14}$ Instituto de Astrofísica de Canarias (IAC), E-38200 La Laguna, Tenerife, Spain \\ ${ }^{15}$ Departamento de Astrofísica, Universidad de La Laguna (ULL), E-38205 La Laguna, Tenerife, Spain \\ ${ }^{16}$ Department of Physics and Astronomy, Siena College, Loudonville, NY 12211, USA \\ ${ }^{17}$ CAS Key Laboratory for Research in Galaxies and Cosmology, Department of Astronomy, University of Science and Technology of China, \\ Hefei, Anhui 230026, China \\ ${ }^{18}$ CEA Saclay, DSM/Irfu/Service d'Astrophysique, Orme des Merisiers, F-91191 Gif-sur-Yvette Cedex, France \\ ${ }^{19}$ Cahill Center for Astronomy and Astrophysics, California Institute of Technology, MC 249-17, Pasadena, CA 91125, USA \\ Received 2015 November 16; accepted 2016 February 7; published 2016 March 24
}

\begin{abstract}
In this paper we present the results of our search for and study of $z \gtrsim 6$ galaxy candidates behind the third Frontier Fields (FFs) cluster, MACS J0717.5+3745, and its parallel field, combining data from Hubble and Spitzer. We select 39 candidates using the Lyman break technique, for which the clear non-detection in optical make the extreme mid- $z$ interlopers hypothesis unlikely. We also take benefit from $z \gtrsim 6$ samples selected using the previous FF data sets of Abell 2744 and MACS 0416 to improve the constraints on the properties of very high redshift objects. We compute the redshift and the physical properties such emission lines properties, star formation rate, reddening, and stellar mass for all FF objects from their spectral energy distribution using templates including nebular emission lines. We study the relationship between several physical properties and confirm the trend already observed in previous surveys for evolution of star formation rate with galaxy mass and between the size and the UV luminosity of our candidates. The analysis of the evolution of the UV luminosity function with redshift seems more compatible with an evolution of density. Moreover, no robust $z \geqslant 8.5$ object is selected behind the cluster field and few $z \sim 9$ candidates have been selected in the two previous data sets from this legacy survey, suggesting a strong evolution in the number density of galaxies between $z \sim 8$ and 9 . Thanks to the use of the lensing cluster, we study the evolution of the star formation rate density produced by galaxies with $L>0.03 L^{\star}$, and confirm the strong decrease observed between $z \sim 8$ and 9 .
\end{abstract}

Key words: cosmology: observations - galaxies: clusters: individual (MACS J0717.5+3745) - galaxies: highredshift - gravitational lensing: strong

\section{INTRODUCTION}

One of the most intriguing challenges of the coming decade is undoubtedly the search for the first stars and galaxies that appeared a few hundreds million years after the Big Bang. During the last ten years, major advances have been made in the quest of the first galaxies in our universe, thanks to the commissioning of new facilities such as the WFC3/Hubble Space Telescope (HST) (Windhorst et al. 2011), WIRCam/CFHT (Puget et al. 2004), MOSFIRE/Keck (McLean et al. 2012) or X-Shooter/VLT (Vernet et al. 2011), and the arrival of extremely deep surveys, e.g., the Hubble Ultra Deep Field (Beckwith et al. 2006), the Cluster Lensing And Supernova survey with Hubble (CLASHPostman et al. 2012), or the Brightest of Reionizing Galaxies

\footnotetext{
${ }^{20}$ Hubble Fellow.
}

Survey (BoRG-Trenti et al. 2011). Among all the results achieved, one can mention the great leap forward in the number of $z \gtrsim 6.5$ sources known that count in several hundreds at $z \sim 7$ (Bouwens et al. 2010; Oesch et al. 2010c; Schenker et al. 2013), hundreds at $z \sim 8$ (Bradley et al. 2012; Yan et al. 2012; Labbé et al. 2013) and dozens at $z \gtrsim 8.5$ (McLure et al. 2013; Oesch et al. 2014), with the most distant spectroscopically confirmed galaxy at $z=8.68$ (Zitrin et al. 2015b) and the highest photometrically selected galaxy at $z \sim 11$ (Coe et al. 2013).

The main interest of studying the first galaxies is to constrain the role they played during the reionization of the universe. This period corresponds to the reionization of neutral hydrogen in the early universe by UV photons (e.g., Zaroubi 2013). The end of this phenomenon is relatively well-defined by observations of quasars at $5.9 \leqslant z \leqslant 6.4$ (Schroeder et al. 2013; 
McGreer et al. 2015). The most likely sources of reionization are primeval galaxies; however, the contribution from galaxies detected in current surveys is not sufficient to match the ionizing background required to reionize the universe at $z \sim 6$ (Madau et al. 1999; Duncan \& Conselice 2015). Recent studies have demonstrated that abundant fainter galaxies below the detection limits of current instruments may have played a crucial role in this process (Bouwens et al. 2015a). One way to start studying these fainter objects before the arrival of extremely large telescopes is to harness gravitational lensing which amplifies their light (Kneib \& Natarajan 2011). Several studies have already demonstrated the interest of using galaxy clusters to detect the faintest objects during the first billion years of the universe (Maizy et al. 2010; Zheng et al. 2012; Zitrin et al. 2015a), but the number of faint sources is not sufficient to give robust constraints on their properties during the epoch of reionization.

The number of relatively bright objects, however, starts to be sufficient to at least study the bright end of the UV luminosity function (LF) and its evolution over the first billion years of the universe. The study of the luminosity distribution of galaxies at lower redshift confirms that the UV LF is well-fitted by a Schechter (1976) function (Cucciati et al. 2012). However, the analysis of several deep blank fields suggests that the bright part of the UV LF at $z>6$ deviates from the standard shape (Bowler et al. 2014; Finkelstein et al. 2015) with an overdensity of bright objects. This could be explained by a decrease of the active galactic nucleus (AGN) feedback that usually suppresses star formation in these galaxies, limiting their growth and thus the number of very massive (and bright) galaxies. If this over-density of bright objects in the early universe is confirmed, it could demonstrate that the role of AGN at such epochs is likely to be less important than at lowredshift (Ilbert et al. 2013) and could be a crucial key to improving our understanding of the reionization process. But other deep blank fields are needed to validate this conclusion.

In 2013 September, the new flagship program of the Hubble Space Telescope, namely the Frontier Fields (FFs), began observations (Lotz et al. 2014). Thanks to the HST design, two fields for each of the six clusters planned for this program are observed simultaneously: one centered on a gravitationally lensed cluster and the second, a "parallel field" located a few arcmins from the main field. The combination of these two types of fields allow us to study the most distant star-forming objects in the early universe over a large range of luminosities. To date, four clusters have been completed (namely Abell 2744, MACS J0416.1-2403, MACS J0717.5+3745, and MACS $1149.5+2223$ ) and the analysis of the first two data sets has already proved the great potential of this project. For example, one of the most distant objects currently known, $(z \sim 10)$, was selected from the FF images and showed multiple images that strongly confirm its photometric redshift (Zitrin et al. 2014). Dozens of objects have already been studied and have led to an improvement of the constraints on the faint end slope of the UV LF (Atek et al. 2014, 2015b; Laporte et al. 2014, 2015; McLeod et al. 2015; Oesch et al. 2015a; Zheng et al. 2014; Ishigaki et al. 2015; Kawamata et al. 2015). More recently, Infante et al. (2015) published the discovery of a strongly amplified $z \sim 10$ candidate $(\mu \sim 20)$ probing, for the first time, the extreme faint end of the UV LF at $z \sim 10$.
In this paper, we present samples selected in MACS J0717.5 +3745 cluster and parallel fields and combine them with similar studies made in Abell 2744 (Zheng et al. 2014; Kawamata et al. 2015) and MACS 0416 (Infante et al. 2015) to obtain a uniform sample and to add robust constraints on the UV LF over the redshift range covered by this legacy program. The organization is as follows. in Section 2 we describe the data set; in Section 4 we discuss the criteria used to select the candidates that are described in Section 5; in Section 6 we estimate the contamination rate of our samples (Section 6.2), computed in the shape of UV LF and the evolution of the SFRd as seen from half of the FF observations (Section 6.4). Throughout this paper, we use a concordance cosmology $\left(\Omega_{M}=0.3, \Omega_{\Lambda}=0.7\right.$ and $\left.H_{0}=70 \mathrm{~km} \mathrm{~s}^{-1} \mathrm{Mpc}^{-1}\right)$ and all magnitudes are quoted in the $A B$ system (Oke \& Gunn 1983).

\section{DATA PROPERTIES}

The FF project is carried out using HST Director's Discretionary Time and will use 840 orbits during Cycles 21, 22 , and 23 with six strong-lensing galaxy clusters as the main targets. For each cluster, the final data set is composed of three images from ACS/HST (F435W, F606W, and F814W) and four images from WFC3/HST (F105W, F125W, F140W, and F160W) reaching depths of $\sim 29$ mag at $5 \sigma$ in a 0.4 diameter aperture. In this study, we used the final data release on MACS J0717.5+3745 $(z=0.551$, Ebeling et al. 2004; Medezinski et al. 2013) made public on 2015 April 1. This third cluster in the FF list has been observed by HST through several observing programs, mainly those related to CLASH (ID: 12103, PI: M. Postman) and the FFs (ID: 13498, PI: J. Lotz). We measured the depth of each image using non-overlapping empty 0 ". 2 radius apertures distributed over the field.

We matched the HST data with deep Spitzer/IRAC images obtained from observations (ID: 90259) carried out from 2013 August to 2015 January combined with archival data from 2007 November to 2013 June. We merged all the raw files using MOPEX tasks and obtained a final image of $449 \mathrm{ks}$ in each band reaching a $5 \sigma$ mag of $A B \sim 25.6$. Table 1 displays exposure time, depth, and filter properties of the data set we used.

\section{SOURCE EXTRACTION}

We used SExtractor (version 2.19.5, Bertin \& Arnouts 1996) to extract sources from our images with extraction parameters defined in Laporte et al. (2015). WFC3 catalogs were built on double image mode using a sum of NIR data as the detection image and then matched to single image mode ACS catalogs with TOPCAT (Taylor 2005) to avoid any false detections at optical wavelengths. Non-detections were measured on the original images whereas colors were measured on psf-matched data using Tiny Tim models (Krist et al. 2011). We measured colors in SExtractor MAG_AUTO apertures defined with Kron_fact $=1.2$ and min_radius $=1.7$ and applied aperture corrections using SExractor MAG_AUTO with default parameters (Kron_fact $=2.5$ and min_radius $=3.5$ ) in the F160W band as reference. Error bars were estimated from the noise measured in several empty 0". 4 diameter apertures distributed around each candidate.

Because we are using extraction parameters defined to select small and faint objects, our catalogs contain several false detections such as pixels in the halos of bright galaxies, pixels in high background level regions, etc. Thus, visual inspection is 
Table 1

Properties of the HST and Spitzer Data

\begin{tabular}{|c|c|c|c|c|c|c|c|}
\hline Filter & $\begin{array}{c}\lambda_{\text {central }} \\
(\mu \mathrm{m})\end{array}$ & $\begin{array}{c}\Delta \lambda \\
(\mathrm{nm})\end{array}$ & Instrument & $\begin{array}{l}t_{\text {exp }}^{C} \\
(\mathrm{ks})\end{array}$ & $\begin{array}{c}m_{C}(5 \sigma) \\
(A B)\end{array}$ & $\begin{array}{l}t_{\exp }^{P} \\
(\mathrm{ks})\end{array}$ & $\begin{array}{c}m_{P}(5 \sigma) \\
(A B)\end{array}$ \\
\hline F435W & 0.431 & 72.9 & ACS & 54.5 & 29.1 & 45.7 & 29.3 \\
\hline F606W & 0.589 & 156.5 & ACS & 33.5 & 29.3 & 25.0 & 29.4 \\
\hline F105W & 1.050 & 300.0 & WFC3 & 67.3 & 28.4 & 79.9 & 28.8 \\
\hline F125W & 1.250 & 300.0 & WFC3 & 33.1 & 28.4 & 34.2 & 28.4 \\
\hline F140W & 1.400 & 400.0 & WFC3 & 27.6 & 28.4 & 34.2 & 28.6 \\
\hline 3.6 & 3.550 & 750.0 & IRAC & 449 & 25.6 & $\ldots$ & $\ldots$ \\
\hline 4.5 & 4.493 & 1015.0 & IRAC & 449 & 25.6 & $\ldots$ & $\ldots$ \\
\hline
\end{tabular}

Note. Columns: (1) filter ID, (2) filter central wavelength, (3) filter FWHM, (4) Instrument, (5, 6) exposure time and $5 \sigma$ depth in a 0.22 radius aperture for $H S T$ data and 1 !" 4 radius aperture for IRAC images for the cluster-centered field, $(7,8)$ same as columns 5 and 6 , but for the parallel field. $P$ stands for parallel field and $C$ stands for cluster field.
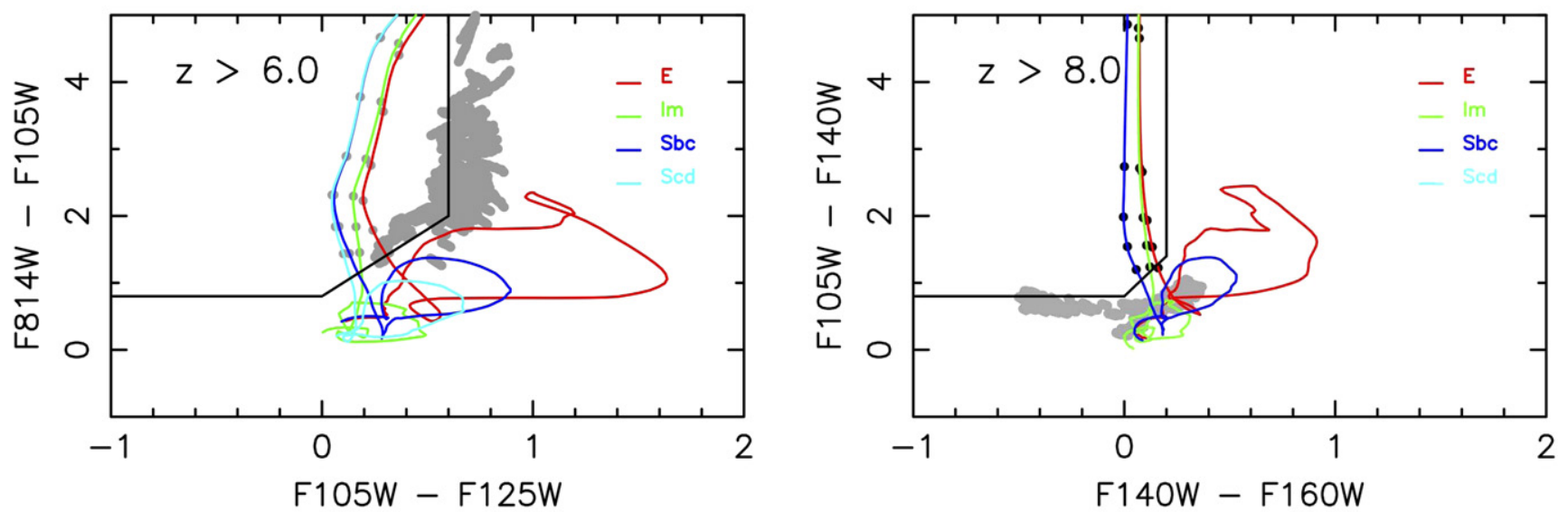

Figure 1. Color criteria we defined to select sources at $z \gtrsim 6$ from the evolution of standard templates (see references in text). Gray dots show the expected colors of objects as a function of redshift starting from $z=6$ on the left panel and $z=8$ on the right panel with a step of $d z=0.2$. The gray dots show the expected colors of $\mathrm{L}$, $\mathrm{M}$, and $\mathrm{T}$ dwarfs from 225 spectra (see references in the text). Our color criteria are shown by the region limited by the black lines.

needed to remove all these non-real sources. We also confirmed the non-detection of all our candidates on optical stacked images.

\section{SELECTION OF HIGH-z CANDIDATES}

One of the most popular methods used to select objects at very high- $z$ in photometric data is the Lyman break technique (Steidel et al. 1999), combining non-detection in images bluewards of the Lyman break and color selection in filters redwards of the break. The selection window was computed using color evolution of standard templates (Coleman et al. 1980; Kinney et al. 1996; Bruzual \& Charlot 2003; Polletta et al. 2007) and defined criteria for several redshift intervals: $z \gtrsim 6$ and $z \gtrsim 8$ (Figure 1 ). To select $z \gtrsim 6$ objects, the color criteria we used are

F814W-F105W $>0.8$

F814W-F105W $>0.8+2.0 \times($ F125W-F140W $)$

F105W-F125W $<0.6$.

The $z \gtrsim 8$ selection criteria are defined as below:

F105W-F140W $>0.8$

F140W-F160W $<0.2$

F105W-F140W $>0.8+3 \times($ F140W-F160W $)$

We used the selection criteria defined by Infante et al. (2015) to select $z \gtrsim 10$ candidates:

$\mathrm{F} 125 \mathrm{~W}-\mathrm{F} 160 \mathrm{~W}>0.8$.
For each redshift interval explored, non-detection criteria are required in all the bands bluewards of the Lyman break, such as $m(\mathrm{~F} 435 \mathrm{~W}, \mathrm{~F} 606 \mathrm{~W}, \mathrm{~F} 814 \mathrm{~W})>m(2 \sigma)$ to select $z \gtrsim 8$ objects. Moreover, to limit spurious selection, we imposed a detection in at least two consecutive bands at more than $5 \sigma$ such as $m(\mathrm{~F} 125 \mathrm{~W}, \mathrm{~F} 140 \mathrm{~W})<m(5 \sigma)$ for $z \gtrsim 8$ objects, leading to a break of at least $\sim 2$ mag that should help to remove extreme mid- $z$ interlopers (Hayes et al. 2012). The reason for such care is that the verification of these techniques holds to $z \sim 5.5$, but has yet to be strongly proved at $z>6-6.5$, and thus the selection of faint candidates without such breaks may be dangerous and lead to an overestimation of the number of objects. We prefer to build a robust sample.

\subsection{Confirming Optical Non-detection: $\chi_{\mathrm{opt}}^{2}$}

The visual inspection of our candidates could still allow objects that are extremely faint in optical bands into our samples and that could not be at such high redshift (see Section 6.2). In order to limit or remove this kind of interloper, we applied the optical $\chi^{2}$ method defined in Bouwens et al. (2011b) by

$$
\chi_{\mathrm{opt}}^{2}=\sum_{i=1}^{n} \operatorname{SGN}\left(f_{i}\right)\left(\frac{f_{i}}{\sigma_{i}}\right)^{2}
$$




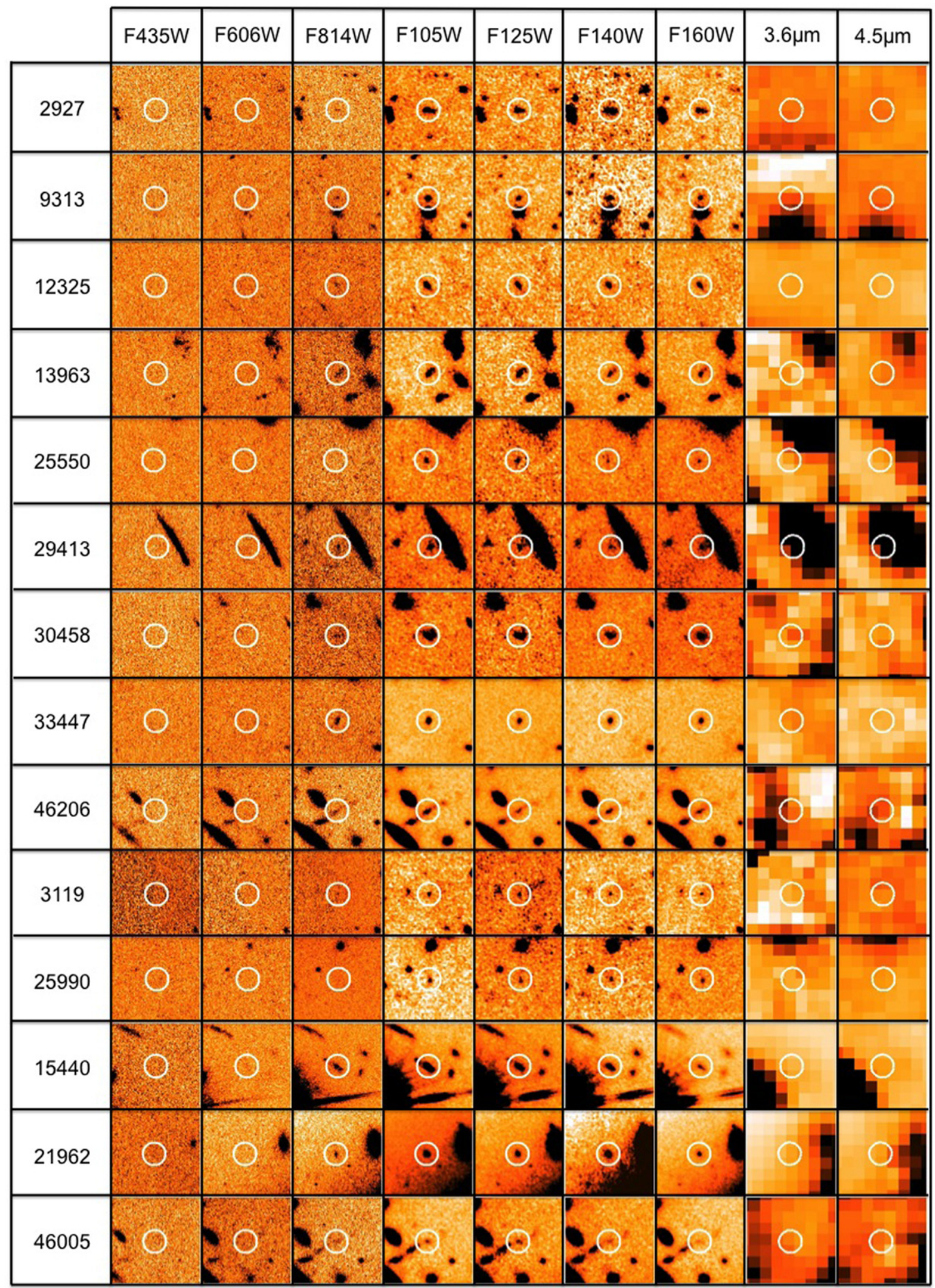

Figure 2. Thumbnail images of $z \sim 7$ candidates selected behind the cluster field. Each stamp is $5^{\prime \prime} \times 5^{\prime \prime}$ and the position of each candidate is displayed as a white $00^{\prime \prime} 6$ radius circle.

where $f_{i}$ is the flux measured in band $i, \sigma_{i}$ is the uncertainty on $f_{i}$ and $\operatorname{SGN}\left(f_{i}\right)=1$ if $f_{i}>0$ or $\operatorname{SGN}\left(f_{i}\right)=-1$ if $f_{i}<0$.

To estimate the $\chi_{\mathrm{opt}}^{2}$ limit above which a candidate should be considered as detected in the optical bands, we measured the optical flux in 1000 empty 0". 4 diameter apertures distributed over the selection area and computed for each aperture the $\chi_{\mathrm{opt}}^{2}$. We then added with the IRAF mkobjects routine sources that are detected at $\sim 2 \sigma$ in the optical bands and computed for each source its $\chi_{\mathrm{opt}}^{2}$. We compared the $\chi_{\mathrm{opt}}^{2}$ distribution for each sample (empty apertures and faint objects) and deduced the 
Table 2

$6 \leqslant z \leqslant 8$ Objects Selected on the Cluster Field

\begin{tabular}{|c|c|c|c|c|c|c|c|c|c|c|}
\hline ID & $\begin{array}{c}\text { R.A. } \\
\text { (J2000) }\end{array}$ & $\begin{array}{c}\text { Decl. } \\
(\mathrm{J} 2000)\end{array}$ & $\begin{array}{c}\mathrm{F} 814 \mathrm{~W} \\
(A B)\end{array}$ & $\begin{array}{c}\text { F105W } \\
(A B)\end{array}$ & $\begin{array}{c}\mathrm{F} 125 \mathrm{~W} \\
(A B)\end{array}$ & $\begin{array}{c}\text { F140W } \\
(A B)\end{array}$ & $\begin{array}{c}\text { F160W } \\
(A B)\end{array}$ & $\begin{array}{c}3.6 \mu \mathrm{m} \\
(A B)\end{array}$ & $\begin{array}{c}4.5 \mu \mathrm{m} \\
(A B)\end{array}$ & $\chi_{\mathrm{opt}}^{2}$ \\
\hline 2927 & 109.3892755 & 37.7248568 & $\begin{array}{l}29.19 \\
\pm 0.21\end{array}$ & $\begin{array}{r}26.87 \\
\pm 0.05\end{array}$ & $\begin{array}{r}26.71 \\
\pm 0.04\end{array}$ & $\begin{array}{r}26.73 \\
\pm 0.05\end{array}$ & $\begin{array}{r}26.74 \\
\pm 0.05\end{array}$ & $\begin{array}{l}25.49 \\
\pm 0.14\end{array}$ & $\begin{array}{l}25.43 \\
\pm 0.10\end{array}$ & -0.38 \\
\hline 9313 & 109.381054 & 37.7316083 & $\begin{array}{l}28.83 \\
\pm 0.15\end{array}$ & $\begin{array}{r}26.91 \\
\pm 0.05\end{array}$ & $\begin{array}{r}26.87 \\
\pm 0.05\end{array}$ & $\begin{array}{r}27.04 \\
\pm 0.06\end{array}$ & $\begin{array}{r}27.16 \\
\pm 0.07\end{array}$ & $\begin{array}{c}\text { blended } \\
\quad \ldots\end{array}$ & $\begin{array}{c}\text { blended } \\
\ldots\end{array}$ & -0.62 \\
\hline 12325 & 109.4136628 & 37.7346385 & $\begin{array}{l}29.61 \\
\pm 0.31\end{array}$ & $\begin{array}{r}26.72 \\
\pm 0.05\end{array}$ & $\begin{array}{r}26.68 \\
\pm 0.04\end{array}$ & $\begin{array}{r}26.81 \\
\pm 0.05\end{array}$ & $\begin{array}{r}26.96 \\
\pm 0.06\end{array}$ & $\begin{array}{c}>26.60 \\
\ldots\end{array}$ & $\begin{array}{c}>26.60 \\
\ldots\end{array}$ & 0.03 \\
\hline 13963 & 109.3770165 & 37.7364332 & $\begin{array}{l}29.29 \\
\pm 0.23\end{array}$ & $\begin{array}{r}26.77 \\
\pm 0.05\end{array}$ & $\begin{array}{r}26.64 \\
\pm 0.04\end{array}$ & $\begin{array}{r}26.86 \\
\pm 0.05\end{array}$ & $\begin{array}{r}26.80 \\
\pm 0.05\end{array}$ & $\begin{array}{c}>26.60 \\
\ldots\end{array}$ & $\begin{array}{c}>26.60 \\
\ldots\end{array}$ & 0.13 \\
\hline 25550 & 109.4159 & 37.7467276 & $\begin{array}{c}>30.2 \\
\ldots\end{array}$ & $\begin{array}{r}27.73 \\
\pm 0.11\end{array}$ & $\begin{array}{r}27.56 \\
\pm 0.10\end{array}$ & $\begin{array}{r}27.83 \\
\pm 0.13\end{array}$ & $\begin{array}{r}27.77 \\
\pm 0.13\end{array}$ & $\begin{array}{c}>26.60 \\
\ldots\end{array}$ & $\begin{array}{c}>26.60 \\
\ldots\end{array}$ & 0.08 \\
\hline 29413 & 109.3814076 & 37.7503301 & $\begin{array}{l}29.09 \\
\pm 0.19\end{array}$ & $\begin{array}{r}26.67 \\
\pm 0.04\end{array}$ & $\begin{array}{r}26.84 \\
\pm 0.05\end{array}$ & $\begin{array}{r}26.64 \\
\pm 0.04\end{array}$ & $\begin{array}{r}26.56 \\
\pm 0.04\end{array}$ & $\begin{array}{c}\text { blended } \\
\quad \ldots\end{array}$ & $\begin{array}{c}\text { blended } \\
\quad \ldots\end{array}$ & 0.09 \\
\hline 30458 & 109.3862351 & 37.7519202 & $\begin{array}{l}28.98 \\
\pm 0.18\end{array}$ & $\begin{array}{r}26.45 \\
\pm 0.04\end{array}$ & $\begin{array}{r}26.30 \\
\pm 0.03\end{array}$ & $\begin{array}{r}26.39 \\
\pm 0.04\end{array}$ & $\begin{array}{r}26.44 \\
\pm 0.04\end{array}$ & $\begin{array}{c}\text { blended } \\
\quad \ldots\end{array}$ & $\begin{array}{c}\text { blended } \\
\ldots\end{array}$ & 0.00 \\
\hline 33447 & 109.4090663 & 37.7546801 & $\begin{array}{l}28.39 \\
\pm 0.10\end{array}$ & $\begin{array}{r}26.21 \\
\pm 0.03\end{array}$ & $\begin{array}{r}26.27 \\
\pm 0.03\end{array}$ & $\begin{array}{r}26.29 \\
\pm 0.03\end{array}$ & $\begin{array}{r}26.41 \\
\pm 0.04\end{array}$ & $\begin{array}{l}25.21 \\
\pm 0.14\end{array}$ & $\begin{array}{l}25.61 \\
\pm 0.13\end{array}$ & -0.14 \\
\hline 46206 & 109.3990963 & 37.7649606 & $\begin{array}{l}29.16 \\
\pm 0.21\end{array}$ & $\begin{array}{l}25.98 \\
\pm 0.02\end{array}$ & $\begin{array}{l}25.79 \\
\pm 0.02\end{array}$ & $\begin{array}{r}25.92 \\
\pm 0.02\end{array}$ & $\begin{array}{r}25.89 \\
\pm 0.02\end{array}$ & $\begin{array}{c}\text { blended } \\
\quad \ldots\end{array}$ & $\begin{array}{c}\text { blended } \\
\quad \ldots\end{array}$ & -0.87 \\
\hline 3119 & 109.3854632 & 37.7251234 & $\begin{array}{l}29.91 \\
\pm 0.41\end{array}$ & $\begin{array}{r}27.87 \\
\pm 0.13\end{array}$ & $\begin{array}{r}28.28 \\
\pm 0.19\end{array}$ & $\begin{array}{r}27.98 \\
\pm 0.15\end{array}$ & $\begin{array}{r}27.69 \\
\pm 0.12\end{array}$ & $\begin{array}{c}>26.60 \\
\ldots\end{array}$ & $\begin{array}{c}>26.60 \\
\ldots\end{array}$ & -1.41 \\
\hline 25990 & 109.3694898 & 37.7470086 & $\begin{array}{l}30.43 \\
\pm 0.67\end{array}$ & $\begin{array}{r}27.83 \\
\pm 0.13\end{array}$ & $\begin{array}{r}28.08 \\
\pm 0.16\end{array}$ & $\begin{array}{r}28.03 \\
\pm 0.16\end{array}$ & $\begin{array}{r}28.00 \\
\pm 0.16\end{array}$ & $\begin{array}{c}>26.60 \\
\ldots\end{array}$ & $\begin{array}{c}>26.60 \\
\ldots\end{array}$ & 0.17 \\
\hline 15440 & 109.39233 & 37.738083 & $\begin{array}{l}27.22 \\
\pm 0.02\end{array}$ & $\begin{array}{r}26.51 \\
\pm 0.01\end{array}$ & $\begin{array}{r}26.60 \\
\pm 0.01\end{array}$ & $\begin{array}{r}26.67 \\
\pm 0.01\end{array}$ & $\begin{array}{r}26.90 \\
\pm 0.01\end{array}$ & $\begin{array}{c}\text { blended } \\
\quad \ldots\end{array}$ & $\begin{array}{c}\text { blended } \\
\ldots\end{array}$ & 0.19 \\
\hline 21962 & 109.40773 & 37.742736 & $\begin{array}{l}27.99 \\
\pm 0.04\end{array}$ & $\begin{array}{r}26.69 \\
\pm 0.01\end{array}$ & $\begin{array}{r}26.74 \\
\pm 0.01\end{array}$ & $\begin{array}{r}26.77 \\
\pm 0.01\end{array}$ & $\begin{array}{r}26.90 \\
\pm 0.01\end{array}$ & $\begin{array}{c}\text { blended } \\
\quad \ldots\end{array}$ & $\begin{array}{c}\text { blended } \\
\ldots\end{array}$ & 0.20 \\
\hline 46005 & 109.3988091 & 37.7650708 & $\begin{array}{l}29.22 \\
\pm 0.22\end{array}$ & $\begin{array}{r}26.86 \\
\pm 0.05\end{array}$ & $\begin{array}{r}26.94 \\
\pm 0.06\end{array}$ & $\begin{array}{r}27.05 \\
\pm 0.07\end{array}$ & $\begin{array}{r}27.08 \\
\pm 0.07\end{array}$ & $\begin{array}{c}>26.60 \\
\ldots\end{array}$ & $\begin{array}{c}>26.60 \\
\cdots\end{array}$ & 0.13 \\
\hline 802 & 109.3864548 & 37.7346659 & $\begin{array}{c}>30.2 \\
\ldots\end{array}$ & $\begin{array}{r}27.09 \\
\pm 0.06\end{array}$ & $\begin{array}{l}27.14 \\
\pm 0.07\end{array}$ & $\begin{array}{r}26.69 \\
\pm 0.05\end{array}$ & $\begin{array}{r}26.73 \\
\pm 0.05\end{array}$ & $\begin{array}{c}>26.60 \\
\ldots\end{array}$ & $\begin{array}{c}>26.60 \\
\ldots\end{array}$ & 0.26 \\
\hline 16621 & 109.4186495 & 37.7387916 & $\begin{array}{l}29.74 \\
\pm 0.35\end{array}$ & $\begin{array}{r}27.86 \\
\pm 0.13\end{array}$ & $\begin{array}{r}28.05 \\
\pm 0.15\end{array}$ & $\begin{array}{r}27.86 \\
\pm 0.14\end{array}$ & $\begin{array}{r}28.24 \\
\pm 0.19\end{array}$ & $\begin{array}{c}>26.60 \\
\ldots\end{array}$ & $\begin{array}{c}>26.60 \\
\quad \ldots\end{array}$ & 0.24 \\
\hline 47376 & 109.4008562 & 37.7662314 & $\begin{array}{l}29.57 \\
\pm 0.30\end{array}$ & $\begin{array}{r}27.95 \\
\pm 0.14\end{array}$ & $\begin{array}{r}27.86 \\
\pm 0.13\end{array}$ & $\begin{array}{r}27.46 \\
\pm 0.10\end{array}$ & $\begin{array}{r}27.81 \\
\pm 0.13\end{array}$ & $\begin{array}{c}>26.60 \\
\ldots\end{array}$ & $\begin{array}{c}>26.60 \\
\ldots\end{array}$ & 0.21 \\
\hline 15756 & 109.3790232 & 37.7383872 & $\begin{array}{l}30.09 \\
\pm 0.49\end{array}$ & $\begin{array}{r}28.07 \\
\pm 0.16\end{array}$ & $\begin{array}{r}28.00 \\
\pm 0.15\end{array}$ & $\begin{array}{r}28.32 \\
\pm 0.21\end{array}$ & $\begin{array}{r}28.20 \\
\pm 0.19\end{array}$ & $\begin{array}{c}>26.60 \\
\ldots\end{array}$ & $\begin{array}{c}>26.60 \\
\ldots\end{array}$ & 0.84 \\
\hline 17265 & 109.3912133 & 37.7391643 & $\begin{array}{l}29.63 \\
\pm 0.32\end{array}$ & $\begin{array}{r}26.90 \\
\pm 0.05\end{array}$ & $\begin{array}{r}26.74 \\
\pm 0.05\end{array}$ & $\begin{array}{r}26.70 \\
\pm 0.05\end{array}$ & $\begin{array}{r}26.73 \\
\pm 0.05\end{array}$ & $\begin{array}{c}\text { blended } \\
\ldots\end{array}$ & $\begin{array}{c}\text { blended } \\
\ldots\end{array}$ & 1.46 \\
\hline 20756 & 109.3776056 & 37.7417947 & $\begin{array}{l}28.73 \\
\pm 0.14\end{array}$ & $\begin{array}{r}26.61 \\
\pm 0.04\end{array}$ & $\begin{array}{r}26.68 \\
\pm 0.04\end{array}$ & $\begin{array}{r}26.37 \\
\pm 0.04\end{array}$ & $\begin{array}{r}26.46 \\
\pm 0.04\end{array}$ & $\begin{array}{c}>26.60 \\
\ldots\end{array}$ & $\begin{array}{c}>26.60 \\
\ldots\end{array}$ & 0.66 \\
\hline 28748 & 109.3854382 & 37.7499249 & $\begin{array}{l}29.16 \\
\pm 0.21\end{array}$ & $\begin{array}{r}27.40 \\
\pm 0.08\end{array}$ & $\begin{array}{r}27.44 \\
\pm 0.09\end{array}$ & $\begin{array}{r}27.23 \\
\pm 0.08\end{array}$ & $\begin{array}{r}27.23 \\
\pm 0.08\end{array}$ & $\begin{array}{c}>26.60 \\
\ldots\end{array}$ & $\begin{array}{c}>26.60 \\
\ldots\end{array}$ & 0.88 \\
\hline 45614 & 109.3950838 & 37.7644073 & $\begin{array}{l}30.20 \\
\pm 0.54\end{array}$ & $\begin{array}{r}27.53 \\
\pm 0.10\end{array}$ & $\begin{array}{r}27.90 \\
\pm 0.13\end{array}$ & $\begin{array}{r}27.32 \\
\pm 0.08\end{array}$ & $\begin{array}{r}27.46 \\
\pm 0.09\end{array}$ & $\begin{array}{c}>26.60 \\
\ldots\end{array}$ & $\begin{array}{c}>26.60 \\
\ldots\end{array}$ & 0.96 \\
\hline 12402 & 109.4128542 & 37.7338042 & $\begin{array}{l}29.70 \\
\pm 0.34\end{array}$ & $\begin{array}{r}26.51 \\
\pm 0.04\end{array}$ & $\begin{array}{r}26.46 \\
\pm 0.04\end{array}$ & $\begin{array}{r}26.65 \\
\pm 0.05\end{array}$ & $\begin{array}{r}26.65 \\
\pm 0.04\end{array}$ & $\begin{array}{l}25.78 \\
\pm 0.42\end{array}$ & $\begin{array}{l}25.59 \\
\pm 0.35\end{array}$ & 0.62 \\
\hline 13806 & 109.3803311 & 37.7366722 & $\begin{array}{l}29.80 \\
\pm 0.37\end{array}$ & $\begin{array}{r}28.19 \\
\pm 0.17\end{array}$ & $\begin{array}{r}28.37 \\
\pm 0.21\end{array}$ & $\begin{array}{r}28.78 \\
\pm 0.32\end{array}$ & $\begin{array}{r}28.41 \\
\pm 0.23\end{array}$ & $\begin{array}{c}>26.60 \\
\ldots\end{array}$ & $\begin{array}{c}>26.60 \\
\ldots\end{array}$ & 0.73 \\
\hline 14977 & 109.4132994 & 37.7374793 & $\begin{array}{l}30.44 \\
\pm 0.68\end{array}$ & $\begin{array}{r}28.07 \\
\pm 0.16\end{array}$ & $\begin{array}{r}28.14 \\
\pm 0.17\end{array}$ & $\begin{array}{r}28.03 \\
\pm 0.16\end{array}$ & $\begin{array}{r}28.29 \\
\pm 0.20\end{array}$ & $\begin{array}{c}>26.60 \\
\ldots\end{array}$ & $\begin{array}{c}>26.60 \\
\ldots\end{array}$ & 1.45 \\
\hline 26338 & 109.3657244 & 37.7474107 & $\begin{array}{c}>30.2 \\
\quad \ldots\end{array}$ & $\begin{array}{r}27.95 \\
\pm 0.14\end{array}$ & $\begin{array}{r}27.87 \\
\pm 0.13\end{array}$ & $\begin{array}{r}28.14 \\
\pm 0.18\end{array}$ & $\begin{array}{r}27.89 \\
\pm 0.14\end{array}$ & $\begin{array}{c}>26.60 \\
\ldots\end{array}$ & $\begin{array}{c}>26.60 \\
\ldots\end{array}$ & 4.56 \\
\hline 28488 & 109.3698122 & 37.7486357 & $\begin{array}{l}30.16 \\
\pm 0.52\end{array}$ & $\begin{array}{r}27.69 \\
\pm 0.11\end{array}$ & $\begin{array}{r}27.33 \\
\pm 0.08\end{array}$ & $\begin{array}{r}27.75 \\
\pm 0.12\end{array}$ & $\begin{array}{l}27.06 \\
\pm 0.07\end{array}$ & $\begin{array}{c}>26.60 \\
\ldots\end{array}$ & $\begin{array}{c}>26.60 \\
\ldots\end{array}$ & 1.21 \\
\hline 45217 & 109.3968464 & 37.7630624 & $\begin{array}{l}28.40 \\
\pm 0.10\end{array}$ & $\begin{array}{r}27.26 \\
\pm 0.07\end{array}$ & $\begin{array}{r}27.37 \\
\pm 0.08\end{array}$ & $\begin{array}{r}27.07 \\
\pm 0.07\end{array}$ & $\begin{array}{r}27.04 \\
\pm 0.06\end{array}$ & $\begin{array}{c}>26.60 \\
\ldots\end{array}$ & $\begin{array}{c}>26.60 \\
\ldots\end{array}$ & 0.33 \\
\hline
\end{tabular}

Note. All error bars are computed from noise measured in 0. " 4 diameter apertures distributed over each object. The last column displays the $\chi_{\mathrm{opt}}^{2}$; all objects above the solid line have a $\chi_{\mathrm{opt}}^{2}$ consistent with a non-detection in optical. 
Table 3

$z \gtrsim 8$ Objects Selected in the Parallel Field

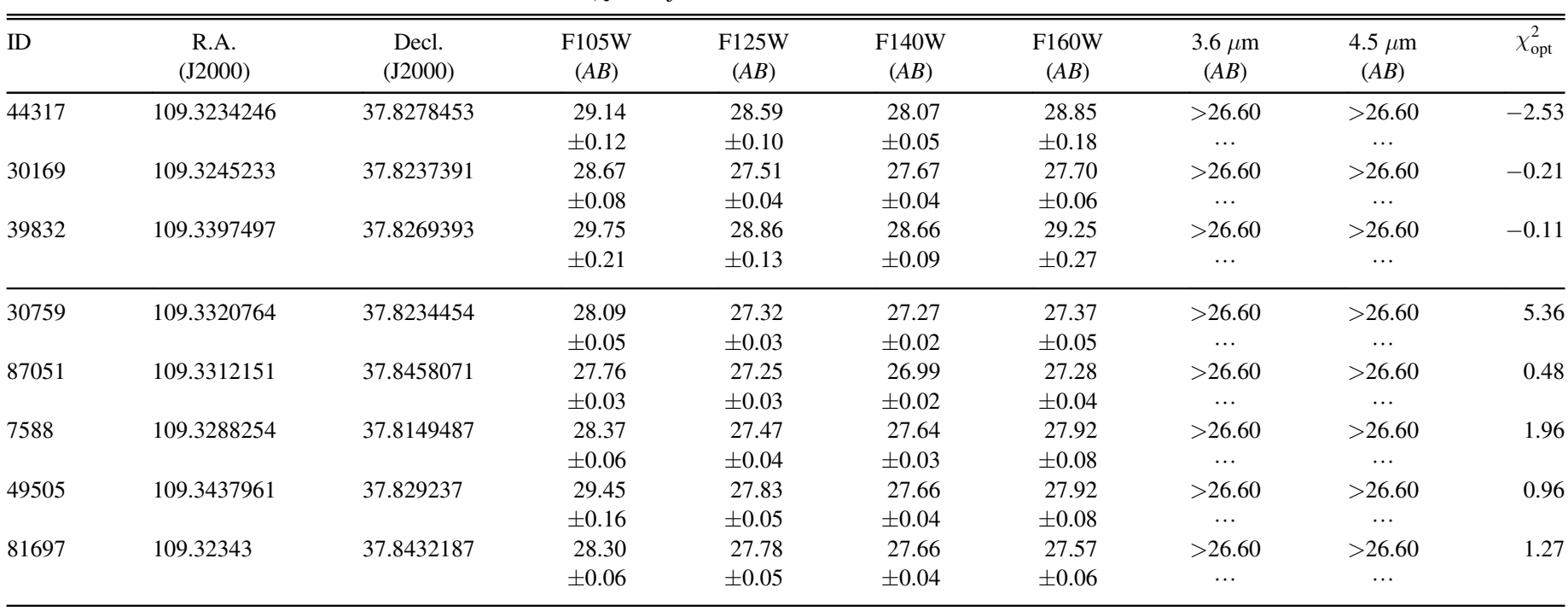

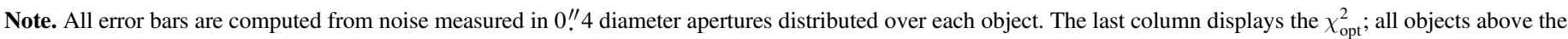
solid line have a $\chi_{\mathrm{opt}}^{2}$ consistent with a non-detection in optical.

$\chi_{\mathrm{opt}}^{2}$ limit from the value where the probability of getting an object with a faint detection in the optical is higher than the probability of getting a non-detected source. We estimated this limit to be 0.2 , therefore all sources with $\chi_{\text {opt }}^{2}>0.2$ will be considered as most likely contaminants. However, because of the intracluster light (DeMaio et al. 2015), we used a $\chi_{\lim }^{2}$ that was a function of the position over the region covered by HST in the cluster field.

Among the $28 z \gtrsim 6$ objects that fulfill the selection criteria in the cluster field, 14 satisfied also the $\chi_{\mathrm{opt}}^{2}$ criteria and are considered to be good candidates in the following. For the parallel field sample, only 25 sources over the 42 selected have a $\chi_{\text {opt }}^{2}$ consistent with a real non-detection. All of these objects, including those considered as detected at optical wavelengths, are presented on Tables 2-4. Thumbnail images of our candidates are shown in Figures 2 and 3.

\subsection{Longer Wavelength Constraints}

We used the deep 3.5 and $4.5 \mu \mathrm{m} /$ IRAC images described in Section 2 to add SED constraints at longer wavelengths. We performed aperture photometry within a circle of 2 " 4 radius and considered all objects for which more than two were inside this aperture as "blended." For the remaining objects we followed the method described in Zheng et al. (2014) using GALFIT (Peng et al. 2010). We then used the aperture correction factors defined in Hora et al. (2008) to obtain the total photometry.

In this way, we can add SED constraints at longer wavelengths for $\approx 72 \%$ of our sample. For the remaining objects, we estimated their physical properties using HST information only (see below).

\section{PROPERTIES OF OUR SAMPLES}

To improve the size of our high- $z$ sample, we combined candidates described in the previous selection with objects selected following the same methods as in the two first FF data sets of Abell 2744 (Zheng et al. 2014; Kawamata et al. 2015) and MACS 0416 (Infante et al. 2015). In the following, we estimate the physical properties of all these objects (redshift,
SFR, stellar mass, reddening, and size) using the same methods to get homogeneous results).

\subsection{Photometric Redshift and Emission Lines}

The SEDs of our candidates are constrained by at least seven measurements (from F435W to F160W) including robust nondetection at short wavelengths. For more than $70 \%$ of our sample, we added constraints on the SEDs within the IRAC wavelength range, making the estimation of their properties more robust. These properties have been deduced by SEDfitting and using two different approaches: $\chi^{2}$ minimization with Hyperz (Bolzonella et al. 2000) ${ }^{21}$ and Bayesian probability with BPZ (Benítez 2000). We run Hyperz with a standard library template, including nebular emission lines (Coleman et al. 1980; Kinney et al. 1996; Fioc \& RoccaVolmerange 1997; Silva et al. 1998; Bruzual \& Charlot 2003; Polletta et al. 2007) and allowing as parameter space $z \in[0.0: 12.0], A_{v} \in[0.0: 3.0] \mathrm{mag}$, following the reddening law defined in Calzetti et al. (2000). Uncertainties on photometric redshift are deduced from the $1 \sigma$ confidence interval (Table 5). BPZ was run spanning a redshift range of $z \in[0.0001,12.0]$ with a resolution of $\Delta z=0.01$, applying no priors to the likelihood functions and using an interpolation factor of 9 among contiguous templates. We used the new library of galaxy models in BPZ2.0 (described in Molino et al. 2014 ) composed by six templates for elliptical galaxies: two for spiral galaxies and four for starburst galaxies along with emission lines and dust extinction. Opacity of the intergalactic medium is applied as described in Madau (1995) for both Hyperz and BPZ.

For most of our sample, photometric redshifts computed from the $\chi^{2}$ minimization method are consistent with those computed using the Bayesian approach, especially for all objects selected in the cluster field. About $\approx 30 \%$ of our candidates have $1 \sigma$ error bars that disagree, but only four objects ( $\approx 10 \%$ of our sample) disagree on the nature of the

\footnotetext{
${ }^{21}$ v12.3 is available at http://userpages.irap.omp.eu/ rpello/newhyperz
} 
Table 4

$6 \leqslant z \leqslant 8$ Objects Selected in the Parallel Field

\begin{tabular}{|c|c|c|c|c|c|c|c|c|c|c|}
\hline ID & $\begin{array}{l}\text { R.A. } \\
\text { (J2000) }\end{array}$ & $\begin{array}{l}\text { Decl. } \\
\text { (J2000) }\end{array}$ & $\begin{array}{c}\mathrm{F} 814 \mathrm{~W} \\
(A B)\end{array}$ & $\begin{array}{c}\mathrm{F} 105 \mathrm{~W} \\
(A B)\end{array}$ & $\begin{array}{c}\text { F125W } \\
(A B)\end{array}$ & $\begin{array}{c}\text { F140W } \\
(A B)\end{array}$ & $\begin{array}{c}\text { F160W } \\
(A B)\end{array}$ & $\begin{array}{c}3.6 \mu \mathrm{m} \\
(A B)\end{array}$ & $\begin{array}{c}4.5 \mu \mathrm{m} \\
(A B)\end{array}$ & $\bar{~} \chi_{\mathrm{opt}}^{2}$ \\
\hline 2035 & 109.3133037 & 37.8101138 & $\begin{array}{c}>30.5 \\
\ldots\end{array}$ & $\begin{array}{r}27.33 \\
\pm 0.02\end{array}$ & $\begin{array}{r}27.07 \\
\pm 0.03\end{array}$ & $\begin{array}{r}27.15 \\
\pm 0.02\end{array}$ & $\begin{array}{r}27.27 \\
\pm 0.04\end{array}$ & $\begin{array}{c}>26.60 \\
\ldots\end{array}$ & $\begin{array}{c}>26.60 \\
\ldots\end{array}$ & -0.09 \\
\hline 6576 & 109.3167913 & 37.8144653 & $\begin{array}{c}>30.5 \\
\ldots\end{array}$ & $\begin{array}{r}28.36 \\
\pm 0.06\end{array}$ & $\begin{array}{l}28.03 \\
\pm 0.06\end{array}$ & $\begin{array}{r}27.92 \\
\pm 0.04\end{array}$ & $\begin{array}{r}27.80 \\
\pm 0.07\end{array}$ & $\begin{array}{c}>26.60 \\
\ldots\end{array}$ & $\begin{array}{c}>26.60 \\
\ldots\end{array}$ & -0.51 \\
\hline 10738 & 109.3218622 & 37.8167184 & $\begin{array}{l}29.51 \\
\pm 0.21\end{array}$ & $\begin{array}{r}27.78 \\
\pm 0.03\end{array}$ & $\begin{array}{c}27.92 \\
\pm 0.06\end{array}$ & $\begin{array}{r}28.19 \\
\pm 0.06\end{array}$ & $\begin{array}{r}27.89 \\
\pm 0.08\end{array}$ & $\begin{array}{c}>26.60 \\
\ldots\end{array}$ & $\begin{array}{c}>26.60 \\
\ldots\end{array}$ & 0.05 \\
\hline 24830 & 109.3045523 & 37.8218034 & $\begin{array}{c}>30.5 \\
\ldots\end{array}$ & $\begin{array}{r}27.36 \\
\pm 0.02\end{array}$ & $\begin{array}{l}27.31 \\
\pm 0.03\end{array}$ & $\begin{array}{r}27.34 \\
\pm 0.03\end{array}$ & $\begin{array}{r}27.20 \\
\pm 0.04\end{array}$ & $\begin{array}{c}>26.60 \\
\ldots\end{array}$ & $\begin{array}{c}>26.60 \\
\ldots\end{array}$ & -0.16 \\
\hline 26762 & 109.3026294 & 37.8226524 & $\begin{array}{c}>30.5 \\
\ldots\end{array}$ & $\begin{array}{r}27.44 \\
\pm 0.03\end{array}$ & $\begin{array}{l}27.71 \\
\pm 0.05\end{array}$ & $\begin{array}{r}27.52 \\
\pm 0.03\end{array}$ & $\begin{array}{r}27.49 \\
\pm 0.05\end{array}$ & $\begin{array}{c}>26.60 \\
\ldots\end{array}$ & $\begin{array}{c}>26.60 \\
\ldots\end{array}$ & -0.15 \\
\hline 32445 & 109.3265426 & 37.8243045 & $\begin{array}{l}29.17 \\
\pm 0.16\end{array}$ & $\begin{array}{r}26.95 \\
\pm 0.02\end{array}$ & $\begin{array}{l}27.02 \\
\pm 0.02\end{array}$ & $\begin{array}{r}27.12 \\
\pm 0.02\end{array}$ & $\begin{array}{r}27.09 \\
\pm 0.04\end{array}$ & $\begin{array}{c}>26.60 \\
\ldots\end{array}$ & $\begin{array}{c}>26.60 \\
\ldots\end{array}$ & -0.43 \\
\hline 33421 & 109.3168345 & 37.8243211 & $\begin{array}{c}>30.5 \\
\ldots\end{array}$ & $\begin{array}{r}27.28 \\
\pm 0.02\end{array}$ & $\begin{array}{r}27.09 \\
\pm 0.03\end{array}$ & $\begin{array}{r}27.23 \\
\pm 0.02\end{array}$ & $\begin{array}{r}27.33 \\
\pm 0.05\end{array}$ & $\begin{array}{c}>26.60 \\
\ldots\end{array}$ & $\begin{array}{c}>26.60 \\
\ldots\end{array}$ & -0.07 \\
\hline 37890 & 109.3431884 & 37.8259591 & $\begin{array}{l}29.58 \\
\pm 0.23\end{array}$ & $\begin{array}{r}27.87 \\
\pm 0.04\end{array}$ & $\begin{array}{r}27.94 \\
\pm 0.06\end{array}$ & $\begin{array}{r}28.11 \\
\pm 0.05\end{array}$ & $\begin{array}{r}28.03 \\
\pm 0.09\end{array}$ & $\begin{array}{c}>26.60 \\
\ldots\end{array}$ & $\begin{array}{c}>26.60 \\
\ldots\end{array}$ & -0.39 \\
\hline 39809 & 109.348495 & 37.8265778 & $\begin{array}{c}>30.5 \\
\ldots\end{array}$ & $\begin{array}{r}27.55 \\
\pm 0.03\end{array}$ & $\begin{array}{l}27.01 \\
\pm 0.02\end{array}$ & $\begin{array}{r}27.07 \\
\pm 0.02\end{array}$ & $\begin{array}{r}27.11 \\
\pm 0.04\end{array}$ & $\begin{array}{c}>26.60 \\
\ldots\end{array}$ & $\begin{array}{c}>26.60 \\
\ldots\end{array}$ & -0.06 \\
\hline 42718 & 109.3511417 & 37.827195 & $\begin{array}{c}>30.5 \\
\ldots\end{array}$ & $\begin{array}{r}26.93 \\
\pm 0.02\end{array}$ & $\begin{array}{l}26.86 \\
\pm 0.02\end{array}$ & $\begin{array}{r}27.03 \\
\pm 0.02\end{array}$ & $\begin{array}{r}27.11 \\
\pm 0.04\end{array}$ & $\begin{array}{c}25.86 \\
0.15\end{array}$ & $\begin{array}{c}27.23 \\
0.74\end{array}$ & -0.07 \\
\hline 43555 & 109.3323968 & 37.8275686 & $\begin{array}{c}>30.5 \\
\ldots\end{array}$ & $\begin{array}{r}27.27 \\
\pm 0.02\end{array}$ & $\begin{array}{l}27.18 \\
\pm 0.03\end{array}$ & $\begin{array}{r}26.98 \\
\pm 0.02\end{array}$ & $\begin{array}{r}26.98 \\
\pm 0.03\end{array}$ & $\begin{array}{c}\text { blended } \\
\quad \ldots\end{array}$ & $\begin{array}{c}\text { blended } \\
\quad \ldots\end{array}$ & -0.11 \\
\hline 46175 & 109.3288319 & 37.828303 & $\begin{array}{c}>30.5 \\
\ldots\end{array}$ & $\begin{array}{r}27.74 \\
\pm 0.03\end{array}$ & $\begin{array}{r}27.30 \\
\pm 0.03\end{array}$ & $\begin{array}{r}27.99 \\
\pm 0.05\end{array}$ & $\begin{array}{r}27.66 \\
\pm 0.06\end{array}$ & $\begin{array}{c}>26.60 \\
\ldots\end{array}$ & $\begin{array}{c}>26.60 \\
\ldots\end{array}$ & -0.06 \\
\hline 46719 & 109.3072346 & 37.8284 & $\begin{array}{c}>30.5 \\
\ldots\end{array}$ & $\begin{array}{r}27.65 \\
\pm 0.03\end{array}$ & $\begin{array}{r}27.84 \\
\pm 0.05\end{array}$ & $\begin{array}{r}28.09 \\
\pm 0.05\end{array}$ & $\begin{array}{r}28.05 \\
\pm 0.09\end{array}$ & $\begin{array}{c}>26.60 \\
\ldots\end{array}$ & $\begin{array}{c}>26.60 \\
\ldots\end{array}$ & 0.02 \\
\hline 50815 & 109.3202576 & 37.8295874 & $\begin{array}{l}29.27 \\
\pm 0.17\end{array}$ & $\begin{array}{r}28.31 \\
\pm 0.06\end{array}$ & $\begin{array}{r}28.26 \\
\pm 0.08\end{array}$ & $\begin{array}{r}27.84 \\
\pm 0.04\end{array}$ & $\begin{array}{r}27.87 \\
\pm 0.07\end{array}$ & $\begin{array}{c}>26.60 \\
\ldots\end{array}$ & $\begin{array}{c}>26.60 \\
\ldots\end{array}$ & -0.27 \\
\hline 58730 & 109.3475986 & 37.8316658 & $\begin{array}{l}29.78 \\
\pm 0.27\end{array}$ & $\begin{array}{r}26.24 \\
\pm 0.01\end{array}$ & $\begin{array}{r}25.99 \\
\pm 0.01\end{array}$ & $\begin{array}{r}26.06 \\
\pm 0.01\end{array}$ & $\begin{array}{r}26.03 \\
\pm 0.01\end{array}$ & $\begin{array}{c}\text { blended } \\
\quad \ldots\end{array}$ & $\begin{array}{c}\text { blended } \\
\quad \ldots\end{array}$ & -1.30 \\
\hline 66722 & 109.3337826 & 37.836336 & $\begin{array}{l}29.76 \\
\pm 0.27\end{array}$ & $\begin{array}{r}28.47 \\
\pm 0.06\end{array}$ & $\begin{array}{r}28.66 \\
\pm 0.11\end{array}$ & $\begin{array}{r}28.97 \\
\pm 0.12\end{array}$ & $\begin{array}{r}28.83 \\
\pm 0.18\end{array}$ & $\begin{array}{c}>26.60 \\
\ldots\end{array}$ & $\begin{array}{c}>26.60 \\
\ldots\end{array}$ & -1.01 \\
\hline 91692 & 109.3254561 & 37.848214 & $\begin{array}{c}>30.5 \\
\ldots\end{array}$ & $\begin{array}{r}28.49 \\
\pm 0.07\end{array}$ & $\begin{array}{r}28.12 \\
\pm 0.07\end{array}$ & $\begin{array}{r}28.26 \\
\pm 0.06\end{array}$ & $\begin{array}{r}28.12 \\
\pm 0.09\end{array}$ & $\begin{array}{c}>26.60 \\
\ldots\end{array}$ & $\begin{array}{c}>26.60 \\
\ldots\end{array}$ & 0.08 \\
\hline 7406 & 109.3273542 & 37.8146525 & $\begin{array}{l}28.84 \\
\pm 0.12\end{array}$ & $\begin{array}{r}26.30 \\
\pm 0.01\end{array}$ & $\begin{array}{r}26.42 \\
\pm 0.01\end{array}$ & $\begin{array}{r}26.24 \\
\pm 0.01\end{array}$ & $\begin{array}{r}26.20 \\
\pm 0.02\end{array}$ & $\begin{array}{c}\text { blended } \\
\quad \ldots\end{array}$ & $\begin{array}{c}\text { blended } \\
\quad \ldots\end{array}$ & -0.06 \\
\hline 17548 & 109.3241209 & 37.8190172 & $\begin{array}{l}28.59 \\
\pm 0.09\end{array}$ & $\begin{array}{r}27.04 \\
\pm 0.02\end{array}$ & $\begin{array}{r}27.10 \\
\pm 0.03\end{array}$ & $\begin{array}{r}27.16 \\
\pm 0.02\end{array}$ & $\begin{array}{r}27.02 \\
\pm 0.03\end{array}$ & $\begin{array}{c}\text { blended } \\
\quad \ldots\end{array}$ & $\begin{array}{c}\text { blended } \\
\quad \ldots\end{array}$ & -0.07 \\
\hline 28313 & 109.3082952 & 37.8231455 & $\begin{array}{l}29.18 \\
\pm 0.16\end{array}$ & $\begin{array}{r}27.35 \\
\pm 0.02\end{array}$ & $\begin{array}{r}27.24 \\
\pm 0.03\end{array}$ & $\begin{array}{r}27.51 \\
\pm 0.03\end{array}$ & $\begin{array}{r}27.45 \\
\pm 0.05\end{array}$ & $\begin{array}{c}>26.60 \\
\ldots\end{array}$ & $\begin{array}{c}>26.60 \\
\ldots\end{array}$ & -0.11 \\
\hline 49274 & 109.3115613 & 37.8291645 & $\begin{array}{c}>30.5 \\
\ldots\end{array}$ & $\begin{array}{r}28.15 \\
\pm 0.05\end{array}$ & $\begin{array}{r}27.92 \\
\pm 0.06\end{array}$ & $\begin{array}{r}28.20 \\
\pm 0.06\end{array}$ & $\begin{array}{r}28.27 \\
\pm 0.11\end{array}$ & $\begin{array}{c}>26.60 \\
\ldots\end{array}$ & $\begin{array}{c}>26.60 \\
\ldots\end{array}$ & -0.29 \\
\hline 70084 & 109.3288391 & 37.8376963 & $\begin{array}{c}>30.5 \\
\ldots\end{array}$ & $\begin{array}{r}27.88 \\
\pm 0.04\end{array}$ & $\begin{array}{l}27.22 \\
\pm 0.03\end{array}$ & $\begin{array}{r}27.32 \\
\pm 0.03\end{array}$ & $\begin{array}{r}26.78 \\
\pm 0.03\end{array}$ & $\begin{array}{c}\text { blended } \\
\ldots\end{array}$ & $\begin{array}{c}\text { blended } \\
\ldots\end{array}$ & -0.40 \\
\hline 3014 & 109.3241258 & 37.8114557 & $\begin{array}{l}29.66 \\
\pm 0.25\end{array}$ & $\begin{array}{r}27.85 \\
\pm 0.04\end{array}$ & $\begin{array}{r}27.61 \\
\pm 0.04\end{array}$ & $\begin{array}{r}27.63 \\
\pm 0.03\end{array}$ & $\begin{array}{r}27.31 \\
\pm 0.04\end{array}$ & $\begin{array}{c}>26.60 \\
\ldots\end{array}$ & $\begin{array}{c}>26.60 \\
\ldots\end{array}$ & 0.65 \\
\hline 58664 & 109.3438555 & 37.8320906 & $\begin{array}{l}29.64 \\
\pm 0.24\end{array}$ & $\begin{array}{r}26.97 \\
\pm 0.02\end{array}$ & $\begin{array}{l}26.99 \\
\pm 0.02\end{array}$ & $\begin{array}{r}27.00 \\
\pm 0.02\end{array}$ & $\begin{array}{r}27.16 \\
\pm 0.04\end{array}$ & $\begin{array}{c}>26.60 \\
\ldots\end{array}$ & $\begin{array}{c}>26.60 \\
\ldots\end{array}$ & 0.44 \\
\hline 51380 & 109.3420084 & 37.8295913 & $\begin{array}{l}29.52 \\
\pm 0.21\end{array}$ & $\begin{array}{r}27.37 \\
\pm 0.02\end{array}$ & $\begin{array}{r}27.34 \\
\pm 0.03\end{array}$ & $\begin{array}{r}27.19 \\
\pm 0.02\end{array}$ & $\begin{array}{l}27.07 \\
\pm 0.04\end{array}$ & $\begin{array}{c}>26.60 \\
\ldots\end{array}$ & $\begin{array}{c}>26.60 \\
\ldots\end{array}$ & 0.39 \\
\hline 32892 & 109.3267033 & 37.8244667 & $\begin{array}{l}29.42 \\
\pm 0.20\end{array}$ & $\begin{array}{r}27.44 \\
\pm 0.03\end{array}$ & $\begin{array}{r}27.37 \\
\pm 0.03\end{array}$ & $\begin{array}{r}27.32 \\
\pm 0.03\end{array}$ & $\begin{array}{r}27.34 \\
\pm 0.05\end{array}$ & $\begin{array}{c}>26.60 \\
\ldots\end{array}$ & $\begin{array}{c}>26.60 \\
\ldots\end{array}$ & 0.53 \\
\hline 32407 & 109.3210662 & 37.8240635 & $\begin{array}{l}29.63 \\
\pm 0.24\end{array}$ & $\begin{array}{r}26.75 \\
\pm 0.01\end{array}$ & $\begin{array}{r}26.35 \\
\pm 0.01\end{array}$ & $\begin{array}{r}26.32 \\
\pm 0.01\end{array}$ & $\begin{array}{r}26.47 \\
\pm 0.02\end{array}$ & $\begin{array}{l}25.55 \\
\pm 0.16\end{array}$ & $\begin{array}{l}25.85 \\
\pm 0.21\end{array}$ & 3.42 \\
\hline 47840 & 109.3335154 & 37.8282783 & $\begin{array}{l}29.97 \\
\pm 0.33\end{array}$ & $\begin{array}{r}28.59 \\
\pm 0.07\end{array}$ & $\begin{array}{r}28.83 \\
\pm 0.13\end{array}$ & $\begin{array}{r}29.14 \\
\pm 0.14\end{array}$ & $\begin{array}{r}28.66 \\
\pm 0.15\end{array}$ & $\begin{array}{c}>26.60 \\
\ldots\end{array}$ & $\begin{array}{c}>26.60 \\
\ldots\end{array}$ & 1.28 \\
\hline 56519 & 109.3428398 & 37.8316497 & $\begin{array}{l}29.36 \\
\pm 0.19\end{array}$ & $\begin{array}{r}28.08 \\
\pm 0.04\end{array}$ & $\begin{array}{r}27.93 \\
\pm 0.06\end{array}$ & $\begin{array}{r}28.14 \\
\pm 0.05\end{array}$ & $\begin{array}{r}28.28 \\
\pm 0.11\end{array}$ & $\begin{array}{c}>26.60 \\
\ldots\end{array}$ & $\begin{array}{c}>26.60 \\
\ldots\end{array}$ & 22.04 \\
\hline 61782 & 109.3227636 & 37.8336724 & $\begin{array}{c}>30.5 \\
\ldots\end{array}$ & $\begin{array}{r}29.26 \\
\pm 0.13\end{array}$ & $\begin{array}{c}28.92 \\
\pm 0.14\end{array}$ & $\begin{array}{r}29.52 \\
\pm 0.20\end{array}$ & $\begin{array}{r}29.16 \\
\pm 0.24\end{array}$ & $\begin{array}{c}>26.60 \\
\ldots\end{array}$ & $\begin{array}{c}>26.60 \\
\ldots\end{array}$ & 1.45 \\
\hline 75653 & 109.3184585 & 37.8402601 & $\begin{array}{l}28.69 \\
\pm 0.10\end{array}$ & $\begin{array}{r}27.38 \\
\pm 0.02\end{array}$ & $\begin{array}{r}27.08 \\
\pm 0.03\end{array}$ & $\begin{array}{r}27.21 \\
\pm 0.02\end{array}$ & $\begin{array}{r}26.99 \\
\pm 0.03\end{array}$ & $\begin{array}{c}>26.60 \\
\ldots\end{array}$ & $\begin{array}{c}>26.60 \\
\ldots\end{array}$ & 1.50 \\
\hline 83324 & 109.30879 & 37.8438485 & $>30.5$ & 27.16 & 26.81 & 26.64 & 26.55 & 26.30 & 26.84 & 2.07 \\
\hline
\end{tabular}


Table 4

(Continued)

\begin{tabular}{|c|c|c|c|c|c|c|c|c|c|c|}
\hline ID & $\begin{array}{l}\text { R.A. } \\
\text { (J2000) }\end{array}$ & $\begin{array}{l}\text { Decl. } \\
\text { (J2000) }\end{array}$ & $\begin{array}{c}\text { F814W } \\
(A B)\end{array}$ & $\begin{array}{c}\mathrm{F} 105 \mathrm{~W} \\
(A B)\end{array}$ & $\begin{array}{c}\mathrm{F} 125 \mathrm{~W} \\
(A B)\end{array}$ & $\begin{array}{c}\text { F140W } \\
(A B)\end{array}$ & $\begin{array}{c}\text { F160W } \\
(A B)\end{array}$ & $\begin{array}{c}3.6 \mu \mathrm{m} \\
(A B)\end{array}$ & $\begin{array}{c}4.5 \mu \mathrm{m} \\
(A B)\end{array}$ & $\chi_{\mathrm{opt}}^{2}$ \\
\hline & & & $\ldots$ & \pm 0.02 & \pm 0.02 & \pm 0.01 & \pm 0.02 & \pm 0.22 & \pm 0.61 & \\
\hline 53875 & 109.3309648 & 37.8302942 & $\begin{array}{l}29.49 \\
\pm 0.21\end{array}$ & $\begin{array}{r}27.47 \\
\pm 0.03\end{array}$ & $\begin{array}{r}27.32 \\
\pm 0.03\end{array}$ & $\begin{array}{r}27.15 \\
+0.02\end{array}$ & $\begin{array}{r}27.17 \\
\pm 0.04\end{array}$ & $\begin{array}{c}>26.60 \\
\ldots\end{array}$ & $\begin{array}{c}>26.60 \\
\ldots\end{array}$ & 5.64 \\
\hline 79925 & 109.3120545 & 37.8423928 & $\begin{array}{c}>30.5 \\
\ldots\end{array}$ & $\begin{array}{r}28.43 \\
\pm 0.06\end{array}$ & $\begin{array}{r}28.37 \\
\pm 0.08\end{array}$ & $\begin{array}{r}28.32 \\
\pm 0.07\end{array}$ & $\begin{array}{r}27.92 \\
\pm 0.08\end{array}$ & $\begin{array}{c}>26.60 \\
\ldots\end{array}$ & $\begin{array}{c}>26.60 \\
\ldots\end{array}$ & 1.87 \\
\hline
\end{tabular}

Note. All error bars are computed from noise measured in 0 ." 4 diameter apertures distributed over each object. The last column displays the $\chi_{\mathrm{opt}}^{2}$, all objects above the solid line have a $\chi_{\text {opt }}^{2}$ consistent with a non-detection in optical.

Table 5

Photometric Redshift Computed in two Ways: $\chi^{2}$ Minimization and Bayesian Approach

\begin{tabular}{|c|c|c|c|c|c|}
\hline ID & $z_{\text {Hyperz }}$ & $1 \sigma$ & $z_{B P Z}$ & $1 \sigma$ & $\mu$ \\
\hline 2927 & 6.38 & [5.88: 6.62] & 6.40 & [6.31: 6.45] & $2.01 \pm 0.25$ \\
\hline 9313 & 6.31 & [6.10: 6.57] & 6.42 & [6.34: 6.48] & $2.22 \pm 0.60$ \\
\hline 12325 & 6.74 & [6.50: 6.96] & 6.69 & [6.60: 6.74] & $6.68 \pm 1.75$ \\
\hline $13963^{\mathrm{a}}$ & 6.53 & [6.32: 6.85] & 6.65 & [6.59: 6.73] & $2.51 \pm 0.84$ \\
\hline 25550 & 6.95 & [5.22: 7.47] & 6.89 & [6.77: 7.09] & $5.17 \pm 1.28$ \\
\hline 29413 & 6.38 & [6.14: 6.55] & 6.44 & [6.35: 6.53] & $3.51 \pm 0.89$ \\
\hline 30458 & 6.53 & [6.35: 6.77] & 6.68 & [6.59: 6.72] & $3.22 \pm 1.00$ \\
\hline $33447^{\mathrm{b}}$ & 6.44 & [6.26: 6.57$]$ & 6.51 & {$[6.45: 6.53]$} & $6.97 \pm 2.16$ \\
\hline 46206 & 6.74 & [6.59: 6.95] & 6.80 & [6.75: 6.87] & $3.11 \pm 0.85$ \\
\hline 3119 & 6.26 & [5.41: 6.77] & 6.34 & [6.03: 6.53] & $1.85 \pm 0.28$ \\
\hline 25990 & 6.47 & [5.57: 7.13] & 6.47 & [6.27: 6.71] & $4.17 \pm 1.09$ \\
\hline 15440 & 5.69 & [5.64: 5.75] & 5.73 & [5.67: 5.75] & $18.7 \pm 7.5$ \\
\hline $21962^{b}$ & 5.98 & [5.89: 6.07$]$ & 6.10 & [6.07: 6.14$]$ & $27.9 \pm 8.8$ \\
\hline 46005 & 6.52 & [6.24: 6.73] & 6.53 & [6.44: 6.59] & $3.06 \pm 0.84$ \\
\hline 44317 & 2.11 & [1.69: 2.27] & 7.45 & [7.10: 7.68$]$ & 1.1 \\
\hline 30169 & 8.02 & [7.82: 8.15$]$ & 7.94 & [7.87: 8.00] & 1.1 \\
\hline 39832 & 8.09 & [6.89: 8.47] & 7.82 & [7.38: 8.05] & 1.1 \\
\hline 2035 & 7.11 & [6.84: 7.29] & 7.25 & [7.10: 7.33$]$ & 1.1 \\
\hline 6576 & 6.88 & [3.02: 7.46$]$ & 6.66 & [6.53: 6.97] & 1.1 \\
\hline 10738 & 6.26 & [6.00: 6.54] & 7.05 & [6.97: 7.09] & 1.1 \\
\hline 24830 & 6.48 & [5.42: 7.00] & 6.93 & [6.78: 7.10$]$ & 1.1 \\
\hline 26762 & 6.29 & [6.00: 6.56] & 6.28 & [6.18: 6.38] & 1.1 \\
\hline 32445 & 6.41 & [6.25: 6.61] & 6.85 & [6.76: 6.91] & 1.1 \\
\hline 33421 & 7.05 & [6.75: 7.15] & 6.70 & [6.60: 6.77] & 1.1 \\
\hline 37890 & 6.21 & [5.91: 6.59] & 6.49 & [6.43: 6.54] & 1.1 \\
\hline 39809 & 7.51 & [7.24: 7.64$]$ & 6.89 & [6.85: 6.97] & 1.1 \\
\hline 42718 & 6.67 & [6.47: 6.93] & 6.28 & [6.18: 6.41] & 1.1 \\
\hline 43555 & 6.23 & [5.74: 6.68] & 7.43 & [7.34: 7.48] & 1.1 \\
\hline 46175 & 7.09 & [6.94: 7.23] & 6.77 & [6.75: 6.85] & 1.1 \\
\hline 46719 & 6.36 & [5.53: 6.57] & 6.84 & [6.78: 6.94] & 1.1 \\
\hline 50815 & 0.89 & [0.00: 1.79$]$ & 6.45 & [6.39: 6.52] & 1.1 \\
\hline 58730 & 7.14 & [6.86: 7.18] & 0.79 & [0.67: 0.93$]$ & 1.1 \\
\hline 66722 & 6.04 & [5.66: 6.49] & 7.02 & [6.80: 7.05] & 1.1 \\
\hline 91692 & 7.27 & [4.15: 7.57] & 6.03 & [5.86: 6.24$]$ & 1.1 \\
\hline 7406 & 6.35 & [6.31: 6.43] & 7.13 & [6.70: 7.24] & 1.1 \\
\hline 17548 & 6.11 & [5.95: 6.25$]$ & 6.52 & {$[6.44: 6.56]$} & 1.1 \\
\hline 28313 & 6.22 & [6.05: 6.57] & 6.20 & [6.13: 6.25] & 1.1 \\
\hline 49274 & 6.98 & [6.47: 7.23] & 6.45 & [6.32: 6.54] & 1.1 \\
\hline 70084 & 4.67 & [4.58: 4.72] & 6.83 & [6.69: 6.93] & 1.1 \\
\hline
\end{tabular}

Notes. Columns: (1) Object ID, (2, 3) photometric redshift from Hyperz with the corresponding $1 \sigma$ error, $(4,5)$ photometric redshift from $B P Z$ with the corresponding $1 \sigma$ error, (6) amplification (for objects selected in a parallel field, we set the amplification at $\mu=1.1$ ).

${ }^{\text {a }}$ Object confirmed by HST-spectroscopy at $z=6.51$ in Schmidt et al. (2016).

${ }^{\mathrm{b}} \mathrm{O}$ bjects confirmed by spectroscopy at $z=6.39$ in Vanzella et al. (2014). candidates (from high- $z$ to low- $z$, \#44317, \#50815, \#58730, and \#70084). We consider these four objects as high- $z$ candidates since they satisfied the color-color selection and they fulfilled the optical $\chi^{2}$ criteria. For the remaining objects, fitted with both approaches as high- $z$, the difference between the $1 \sigma$ confidence interval is not surprising regarding the redshift range of our objects

By adopting templates that include nebular emission lines, we can estimate the equivalent width of the $\left[\mathrm{O}_{\mathrm{III}}\right]$ and $\mathrm{H} \beta$ lines and compare these values to what has been previously found at such high-redshifts to check the quality of our SED-fitting results. Figure 4 shows the distribution of the $z \sim 7 \mathrm{FF}$ candidates compared with the distribution of the $20 z \sim 7$ Lyman break galaxies discussed in Smit et al. (2015). The equivalent widths of the $[\mathrm{O} \mathrm{III}]$ and $\mathrm{H} \beta$ lines measured in $\mathrm{FF}$ candidates are in excellent agreement with what has been estimated in Smit et al. (2015), Roberts-Borsani et al. (2015) and Labbé et al. (2013)

\subsection{Magnification}

Within the framework of the FF project, several groups have provided amplification maps that were built using different assumptions on mass models (Merten et al. 2011; Johnson et al. 2014; Richard et al. 2014; Grillo et al. 2015; Zitrin et al. 2015a).

We estimated the amplification of our candidates by averaging all of these models and uncertainties from the standard deviation. All of the objects selected in the cluster field have magnification ranging from 1.8 to 7.0 (Table 5). The parallel field cannot be considered a real blank field since the cluster mass still plays a role on the light amplification at such distances from the cluster core. Among all of the models, only one covers the parallel field (Merten et al. 2011), but with a low resolution. According to this model, we fixed the amplification of the candidates selected in the parallel field to $\mu=1.1$.

We computed the effective surface covered by the three first FF clusters using the amplification map released by the CATS team matched to our detection images. We then masked all the bright objects in the field and computed for each amplification range the area effectively covered by the data. We estimated an effective surface covered by the 3 first FF data set of $\approx 16$ $\operatorname{arcmin}^{2}$.

We also used LENSTOOL (Kneib et al. 1996; Jullo et al. 2007; Jullo \& Kneib 2009) to search for multiple images of our candidates using the CATS models (Richard et al. 2014). According to this model, 10 objects among our samples could 
Table 6

Physical Properties of Candidates Selected on MACS 0717 Data

\begin{tabular}{|c|c|c|c|c|c|c|}
\hline Galaxy ID & Redshift & $\begin{array}{c}\log M_{*} \\
\left(M_{\odot}\right)\end{array}$ & $\begin{array}{r}\log \mathrm{SFR} \\
\left(M_{\odot} \mathrm{yr}^{-1}\right)\end{array}$ & $\begin{array}{l}\text { AGE } \\
(\mathrm{Gyr})\end{array}$ & $A_{v}$ & Cluster \\
\hline 2035 & 7.05 & $8.5_{-0.3}^{+0.2}$ & $0.5_{-0.1}^{+0.1}$ & $0.2_{-0.1}^{+0.3}$ & $0.1_{-0.1}^{+0.1}$ & M0717 \\
\hline 6576 & 6.93 & $8.8_{-0.3}^{+0.3}$ & $0.6_{-0.2}^{+0.2}$ & $0.4_{-0.3}^{+0.3}$ & $0.6_{-0.3}^{+0.5}$ & M0717 \\
\hline 10738 & 6.28 & $8.2_{-0.4}^{+0.3}$ & $0.1_{-0.1}^{+0.1}$ & $0.2_{-0.2}^{+0.3}$ & $0.1_{-0.1}^{+0.1}$ & M0717 \\
\hline 24830 & 6.85 & $8.6_{-0.1}^{+0.1}$ & $1.1_{-0.3}^{+0.3}$ & $0.1_{-0.1}^{+0.2}$ & $1.2_{-0.4}^{+0.4}$ & M0717 \\
\hline 26762 & 6.70 & $9.2_{-0.2}^{+0.1}$ & $0.4_{-2.1}^{+0.3}$ & $0.4_{-0.3}^{+0.3}$ & $0.8_{-0.6}^{+0.6}$ & M0717 \\
\hline 32445 & 6.49 & $8.4_{-0.3}^{+0.2}$ & $0.5_{-0.1}^{+0.1}$ & $0.2_{-0.1}^{+0.3}$ & $0.1_{-0.1}^{+0.0}$ & M0717 \\
\hline 33421 & 6.89 & $8.7_{-0.1}^{+0.1}$ & $0.5_{-0.1}^{+0.1}$ & $0.3_{-0.2}^{+0.3}$ & $0.1_{-0.1}^{+0.1}$ & M0717 \\
\hline 37890 & 6.28 & $8.3_{-0.4}^{+0.3}$ & $0.2_{-0.1}^{+0.2}$ & $0.3_{-0.2}^{+0.3}$ & $0.1_{-0.1}^{+0.1}$ & M0717 \\
\hline 39809 & 7.43 & $8.7_{-0.2}^{+0.2}$ & $0.6_{-0.1}^{+0.1}$ & $0.3_{-0.2}^{+0.2}$ & $0.1_{-0.1}^{+0.1}$ & M0717 \\
\hline 42718 & 6.77 & $9.2_{-0.1}^{+0.1}$ & $-2.8_{-2.9}^{+1.4}$ & $0.1_{-0.1}^{+0.1}$ & $0.2_{-0.1}^{+0.3}$ & M0717 \\
\hline 43555 & 6.60 & $9.5_{-0.1}^{+0.1}$ & $-1.7_{-0.1}^{+0.1}$ & $0.2_{-0.1}^{+0.1}$ & $0.2_{-0.1}^{+0.1}$ & M0717 \\
\hline 46175 & 6.84 & $8.6_{-0.1}^{+0.2}$ & $0.4_{-0.2}^{+0.4}$ & $0.3_{-0.2}^{+0.3}$ & $0.2_{-0.1}^{+1.0}$ & M0717 \\
\hline 46719 & 6.45 & $9.3_{-0.1}^{+0.1}$ & $-1.9_{-0.2}^{+0.1}$ & $0.3_{-0.1}^{+0.1}$ & $0.1_{-0.1}^{+0.3}$ & M0717 \\
\hline 50815 & 7.79 & $8.3_{-0.5}^{+0.1}$ & $0.5_{-0.1}^{+0.4}$ & $0.1_{-0.1}^{+0.3}$ & $0.2_{-0.1}^{+0.1}$ & M0717 \\
\hline 58730 & 7.02 & $8.9_{-0.1}^{+0.1}$ & $1.0_{-0.1}^{+0.1}$ & $0.2_{-0.1}^{+0.1}$ & $0.1_{-0.1}^{+0.1}$ & M0717 \\
\hline 66722 & 6.03 & $8.1_{-0.4}^{+0.3}$ & $-0.1_{-0.1}^{+0.2}$ & $0.4_{-0.3}^{+0.3}$ & $0.2_{-0.1}^{+0.3}$ & M0717 \\
\hline 91692 & 7.13 & $8.5_{-0.3}^{+0.3}$ & $0.3_{-0.2}^{+0.2}$ & $0.3_{-0.2}^{+0.3}$ & $0.3_{-0.2}^{+0.5}$ & M0717 \\
\hline 7406 & 6.52 & $8.4_{-0.2}^{+0.4}$ & $1.1_{-0.2}^{+0.3}$ & $0.1_{-0.1}^{+0.2}$ & $0.2_{-0.1}^{+0.1}$ & M0717 \\
\hline 17548 & 6.20 & $8.6_{-0.3}^{+0.2}$ & $0.5_{-0.1}^{+0.1}$ & $0.3_{-0.2}^{+0.3}$ & $0.1_{-0.1}^{+0.1}$ & M0717 \\
\hline 28313 & 6.45 & $8.4_{-0.4}^{+0.2}$ & $0.4_{-0.1}^{+0.1}$ & $0.2_{-0.2}^{+0.3}$ & $0.1_{-0.1}^{+0.1}$ & M0717 \\
\hline 49274 & 6.83 & $8.3_{-0.3}^{+0.3}$ & $0.2_{-0.1}^{+0.1}$ & $0.3_{-0.2}^{+0.3}$ & $0.1_{-0.1}^{+0.2}$ & M0717 \\
\hline 70084 & 7.41 & $9.0_{-0.4}^{+0.3}$ & $1.2_{-0.2}^{+0.3}$ & $0.2_{-0.1}^{+0.3}$ & $0.7_{-0.2}^{+0.3}$ & M0717 \\
\hline 44317 & 7.45 & $8.7_{-0.3}^{+0.3}$ & $0.4_{-0.2}^{+0.3}$ & $0.4_{-0.2}^{+0.2}$ & $0.6_{-0.3}^{+0.5}$ & M0717 \\
\hline 30169 & 7.94 & $8.6_{-0.3}^{+0.2}$ & $0.5_{-0.1}^{+0.2}$ & $0.3_{-0.2}^{+0.2}$ & $0.2_{-0.1}^{+0.4}$ & M0717 \\
\hline 39832 & 7.82 & $8.4_{-0.3}^{+0.3}$ & $0.2_{-0.2}^{+0.3}$ & $0.3_{-0.2}^{+0.2}$ & $0.5_{-0.3}^{+0.5}$ & M0717 \\
\hline 2927 & 6.40 & $8.8_{-0.1}^{+0.1}$ & $0.5_{-0.1}^{+0.1}$ & $0.3_{-0.2}^{+0.3}$ & $0.4_{-0.2}^{+0.3}$ & M0717 \\
\hline 9313 & 6.42 & $8.4_{-0.1}^{+0.1}$ & $0.3_{-0.2}^{+0.2}$ & $0.3_{-0.2}^{+0.3}$ & $0.1_{-0.1}^{+0.1}$ & M0717 \\
\hline 12325 & 6.69 & $7.9_{-0.4}^{+0.5}$ & $-0.1_{-0.7}^{+0.7}$ & $0.2_{-0.2}^{+0.3}$ & $0.1_{-0.1}^{+0.1}$ & M0717 \\
\hline 13963 & 6.65 & $8.4_{-0.1}^{+0.1}$ & $0.3_{-0.3}^{+0.2}$ & $0.3_{-0.2}^{+0.3}$ & $0.1_{-0.1}^{+0.1}$ & M0717 \\
\hline 25550 & 6.89 & $8.0_{-0.3}^{+0.1}$ & $-0.2_{-0.5}^{+0.4}$ & $0.3_{-0.2}^{+0.3}$ & $0.3_{-0.2}^{+0.5}$ & M0717 \\
\hline 29413 & 6.44 & $8.5_{-0.3}^{+0.3}$ & $0.3_{-0.4}^{+0.3}$ & $0.3_{-0.2}^{+0.3}$ & $0.3_{-0.1}^{+0.3}$ & M0717 \\
\hline 30458 & 6.68 & $8.4_{-0.2}^{+0.3}$ & $0.4_{-0.4}^{+0.4}$ & $0.2_{-0.2}^{+0.3}$ & $0.1_{-0.1}^{+0.1}$ & M0717 \\
\hline 33447 & 6.51 & $8.0_{-0.5}^{+0.6}$ & $0.1_{-0.7}^{+0.7}$ & $0.2_{-0.1}^{+0.3}$ & $0.1_{-0.1}^{+0.1}$ & M0717 \\
\hline 46206 & 6.80 & $8.3_{-0.3}^{+0.3}$ & $0.6_{-0.4}^{+0.4}$ & $0.1_{-0.1}^{+0.1}$ & $0.1_{-0.1}^{+0.1}$ & M0717 \\
\hline 3119 & 6.34 & $8.5_{-0.2}^{+0.1}$ & $0.2_{-0.1}^{+0.1}$ & $0.4_{-0.3}^{+0.3}$ & $0.5_{-0.3}^{+0.5}$ & M0717 \\
\hline 25990 & 6.47 & $8.1_{-0.2}^{+0.3}$ & $-0.2_{-0.4}^{+0.3}$ & $0.4_{-0.2}^{+0.3}$ & $0.4_{-0.3}^{+0.5}$ & M0717 \\
\hline 15440 & 5.73 & $7.6_{-1.3}^{+1.3}$ & $-1.7_{-1.3}^{+1.3}$ & $0.1_{-0.1}^{+0.1}$ & $0.1_{-0.1}^{+0.1}$ & M0717 \\
\hline 21962 & 6.10 & $7.0_{-1.3}^{+1.2}$ & $-0.8_{-1.4}^{+1.4}$ & $0.1_{-0.1}^{+0.1}$ & $0.1_{-0.1}^{+0.1}$ & M0717 \\
\hline 46005 & 6.53 & $8.2_{-0.1}^{+0.2}$ & $0.1_{-0.4}^{+0.3}$ & $0.3_{-0.2}^{+0.3}$ & $0.1_{-0.1}^{+0.1}$ & M0717 \\
\hline
\end{tabular}

Note. The following quantities are reported: ID (1), photo- $z$ (2), $\log \left(M_{\star}\right)(3), \log (\mathrm{SFR})(4)$, age (5), $A_{v}(6)$, and FF (7).

All values are corrected by their magnification factor and errors are shown at the $1 \sigma$ confidence level.

be multiple imaged (\#9313, \#13963, \#21962, \#25550, \#25990. \#29413, \#30458, \#33447, \#46005, and \#46206), but none of these images are detected on FF data; most of them are outside of the field of view covered by HST and several are located at the positions of bight objects on the region covered by FF data. Moreover, we noticed that Vanzella et al. (2014) found that \#13963, \#21962, and \#33447 are multiple images of the same source at $z=6.4$.

\subsection{Stellar Mass, Age, Reddening, UV Slope, and SFR}

We used iSEDfit code (Moustakas et al. 2013) and followed the method described in Infante et al. (2015) to generate 100,000 models including dust and nebular emission lines, assuming an initial mass function from 0.1 to $100 M_{\odot}$. Uniform priors were adopted to estimate the following parameters: the stellar metallicity, the galaxy age, the star formation timescale, and the rest-frame V-band attenuation. We fix the redshift to the Bayesian value given by BPZ. The results are reported on Tables 6 and 7 and example results from iSEDfit are shown in Figure 5. Error bars on each physical parameter included the uncertainties in magnification, which we estimated from all models available for FF clusters (see Section 5.2).

We used the large sample of $z \sim 7$ and eight candidates identified on FF images to study the relationship between the SFR and the galaxy mass that has been extensively investigated at lower redshift (e.g., Davé 2008; Maraston et al. 2010; Curtis- 
Table 7

Physical Properties of all Candidates Selected in the First Three Frontier Fields

\begin{tabular}{|c|c|c|c|c|c|c|c|}
\hline Galaxy ID & Redshift & $\begin{array}{c}\log M_{*} \\
\left(M_{\odot}\right)\end{array}$ & $\begin{array}{r}\log \mathrm{SFR} \\
\left(M_{\odot} \mathrm{yr}^{-1}\right)\end{array}$ & $\begin{array}{l}\text { AGE } \\
\text { (Gyr) }\end{array}$ & $A_{v}$ & Cluster & References \\
\hline YD1 & 8.64 & $8.8_{-0.2}^{+0.3}$ & $0.7_{-0.1}^{+0.2}$ & $0.3_{-0.2}^{+0.2}$ & $0.6_{-0.3}^{+0.5}$ & A2744 & Zheng et al. (2014) \\
\hline YD2 & 8.26 & $8.6_{-0.2}^{+0.2}$ & $0.4_{-0.1}^{+0.1}$ & $0.3_{-0.2}^{+0.2}$ & $0.5_{-0.3}^{+0.5}$ & A2744 & Zheng et al. (2014) \\
\hline YD3 & 8.56 & $8.3_{-0.1}^{+0.1}$ & $0.1_{-0.2}^{+0.1}$ & $0.3_{-0.2}^{+0.2}$ & $0.6_{-0.3}^{+0.5}$ & A2744 & Zheng et al. (2014) \\
\hline YD4 & 8.51 & $9.4_{-0.2}^{+0.1}$ & $1.2_{-0.1}^{+0.1}$ & $0.3_{-0.2}^{+0.2}$ & $0.6_{-0.3}^{+0.4}$ & A2744 & Zheng et al. (2014) \\
\hline YD5 & 8.45 & $8.4_{-0.1}^{+0.1}$ & $0.3_{-0.2}^{+0.1}$ & $0.3_{-0.2}^{+0.2}$ & $0.6_{-0.3}^{+0.5}$ & A2744 & Zheng et al. (2014) \\
\hline YD6 & 8.25 & $9.5_{-0.2}^{+0.2}$ & $1.2_{-0.2}^{+0.3}$ & $0.4_{-0.2}^{+0.2}$ & $0.9_{-0.4}^{+0.5}$ & A2744 & Zheng et al. (2014) \\
\hline YD7 & 8.24 & $9.1_{-0.1}^{+0.1}$ & $1.1_{-0.1}^{+0.1}$ & $0.2_{-0.2}^{+0.2}$ & $0.3_{-0.1}^{+0.2}$ & A2744 & Zheng et al. (2014) \\
\hline YD8 & 8.15 & $8.8_{-0.1}^{+0.1}$ & $0.8_{-0.1}^{+0.1}$ & $0.2_{-0.2}^{+0.2}$ & $0.3_{-0.1}^{+0.3}$ & A2744 & Zheng et al. (2014) \\
\hline YD9 & 8.26 & $8.4_{-0.2}^{+0.2}$ & $0.3_{-0.1}^{+0.1}$ & $0.3_{-0.2}^{+0.2}$ & $0.5_{-0.3}^{+0.5}$ & A2744 & Zheng et al. (2014) \\
\hline YD10 & 8.25 & $8.7_{-0.2}^{+0.2}$ & $0.6_{-0.1}^{+0.1}$ & $0.3_{-0.2}^{+0.2}$ & $0.5_{-0.3}^{+0.5}$ & A2744 & Zheng et al. (2014) \\
\hline YD11 & 8.27 & $8.0_{-0.2}^{+0.2}$ & $-0.1_{-0.3}^{+0.2}$ & $0.3_{-0.2}^{+0.2}$ & $0.7_{-0.3}^{+0.5}$ & A2744 & Zheng et al. (2014) \\
\hline ZD1 & 8.67 & $8.6_{-0.2}^{+0.3}$ & $0.5_{-0.1}^{+0.2}$ & $0.3_{-0.2}^{+0.2}$ & $0.6_{-0.3}^{+0.6}$ & A2744 & Zheng et al. (2014) \\
\hline $\mathrm{ZD} 2$ & 7.85 & $9.4_{-0.2}^{+0.1}$ & $1.4_{-0.1}^{+0.1}$ & $0.2_{-0.1}^{+0.2}$ & $0.4_{-0.1}^{+0.2}$ & A2744 & Zheng et al. (2014) \\
\hline ZD3 & 7.70 & $9.2_{-0.2}^{+0.2}$ & $1.1_{-0.1}^{+0.1}$ & $0.3_{-0.2}^{+0.2}$ & $0.5_{-0.2}^{+0.4}$ & A2744 & Zheng et al. (2014) \\
\hline ZD4 & 7.83 & $8.7_{-0.3}^{+0.3}$ & $0.5_{-0.1}^{+0.2}$ & $0.3_{-0.2}^{+0.2}$ & $0.6_{-0.3}^{+0.5}$ & A2744 & Zheng et al. (2014) \\
\hline ZD5 & 7.63 & $8.6_{-0.2}^{+0.1}$ & $0.4_{-0.1}^{+0.1}$ & $0.3_{-0.2}^{+0.3}$ & $0.3_{-0.2}^{+0.4}$ & A2744 & Zheng et al. (2014) \\
\hline ZD6 & 7.48 & $9.3_{-0.3}^{+0.2}$ & $1.2_{-0.1}^{+0.2}$ & $0.3_{-0.2}^{+0.3}$ & $0.7_{-0.2}^{+0.4}$ & A2744 & Zheng et al. (2014) \\
\hline ZD7 & 7.32 & $8.2_{-0.4}^{+0.5}$ & $0.1_{-0.6}^{+0.5}$ & $0.3_{-0.2}^{+0.2}$ & $0.3_{-0.1}^{+0.4}$ & A2744 & Zheng et al. (2014) \\
\hline ZD8 & 7.52 & $7.5_{-0.8}^{+0.8}$ & $-0.7_{-0.9}^{+0.8}$ & $0.3_{-0.2}^{+0.2}$ & $0.5_{-0.3}^{+0.5}$ & A2744 & Zheng et al. (2014) \\
\hline ZD9 & 6.93 & $8.4_{-0.2}^{+0.3}$ & $0.4_{-0.4}^{+0.3}$ & $0.2_{-0.2}^{+0.3}$ & $0.3_{-0.1}^{+0.2}$ & A2744 & Zheng et al. (2014) \\
\hline ZD10 & 6.86 & $7.5_{-0.6}^{+0.6}$ & $-0.8_{-0.7}^{+0.7}$ & $0.4_{-0.3}^{+0.3}$ & $0.5_{-0.3}^{+0.5}$ & A2744 & Zheng et al. (2014) \\
\hline ZD11 & 6.96 & $7.8_{-0.3}^{+0.4}$ & $-0.2_{-0.6}^{+0.5}$ & $0.2_{-0.2}^{+0.3}$ & $0.1_{-0.1}^{+0.1}$ & A2744 & Zheng et al. (2014) \\
\hline HFF1P-i1 & 7.29 & $8.8_{-0.3}^{+0.3}$ & $0.8_{-0.1}^{+0.1}$ & $0.2_{-0.2}^{+0.3}$ & $0.1_{-0.1}^{+0.1}$ & A2744 & Kawamata et al. (2015) \\
\hline HFF1P-i2 & 6.36 & $8.6_{-0.4}^{+0.3}$ & $0.5_{-0.1}^{+0.1}$ & $0.2_{-0.2}^{+0.3}$ & $0.1_{-0.1}^{+0.1}$ & A2744 & Kawamata et al. (2015) \\
\hline HFF1P-i3 & 6.16 & $9.0_{-0.3}^{+0.2}$ & $0.8_{-0.2}^{+0.2}$ & $0.4_{-0.2}^{+0.3}$ & $0.5_{-0.2}^{+0.4}$ & A2744 & Kawamata et al. (2015) \\
\hline HFF1P-i4 & 7.10 & $8.9_{-0.3}^{+0.3}$ & $0.7_{-0.2}^{+0.2}$ & $0.3_{-0.2}^{+0.3}$ & $0.3_{-0.2}^{+0.4}$ & A2744 & Kawamata et al. (2015) \\
\hline HFF1P- i5 & 7.85 & $8.3_{-0.3}^{+0.5}$ & $0.9_{-0.2}^{+0.5}$ & $0.1_{-0.1}^{+0.2}$ & $0.1_{-0.1}^{+0.1}$ & A2744 & Kawamata et al. (2015) \\
\hline HFF1P-i6 & 6.70 & $8.7_{-0.4}^{+0.3}$ & $0.5_{-0.1}^{+0.2}$ & $0.3_{-0.2}^{+0.3}$ & $0.1_{-0.1}^{+0.2}$ & A2744 & Kawamata et al. (2015) \\
\hline HFF1P-i7 & 6.83 & $8.7_{-0.4}^{+0.3}$ & $0.5_{-0.1}^{+0.2}$ & $0.3_{-0.2}^{+0.3}$ & $0.2_{-0.1}^{+0.3}$ & A2744 & Kawamata et al. (2015) \\
\hline HFF1P-i8 & 6.06 & $8.6_{-0.4}^{+0.3}$ & $0.4_{-0.1}^{+0.1}$ & $0.3_{-0.2}^{+0.4}$ & $0.1_{-0.1}^{+0.2}$ & A2744 & Kawamata et al. (2015) \\
\hline HFF1P-i9 & 6.62 & $8.6_{-0.4}^{+0.3}$ & $0.5_{-0.1}^{+0.2}$ & $0.3_{-0.2}^{+0.3}$ & $0.2_{-0.1}^{+0.2}$ & A2744 & Kawamata et al. (2015) \\
\hline HFF1P-i10 & 5.98 & $8.8_{-0.4}^{+0.3}$ & $0.5_{-0.2}^{+0.2}$ & $0.4_{-0.3}^{+0.3}$ & $0.3_{-0.2}^{+0.4}$ & A2744 & Kawamata et al. (2015) \\
\hline HFF1P-i11 & 5.86 & $8.7_{-0.4}^{+0.3}$ & $0.4_{-0.2}^{+0.2}$ & $0.4_{-0.3}^{+0.3}$ & $0.4_{-0.2}^{+0.5}$ & A2744 & Kawamata et al. (2015) \\
\hline HFF1P-i12 & 7.81 & $8.8_{-0.5}^{+0.3}$ & $0.8_{-0.2}^{+0.3}$ & $0.2_{-0.2}^{+0.3}$ & $0.3_{-0.2}^{+0.4}$ & A2744 & Kawamata et al. (2015) \\
\hline HFF1P-i13 & 6.45 & $8.8_{-0.4}^{+0.3}$ & $0.5_{-0.2}^{+0.3}$ & $0.4_{-0.3}^{+0.3}$ & $0.4_{-0.2}^{+0.5}$ & A2744 & Kawamata et al. (2015) \\
\hline HFF1P-i14 & 5.86 & $8.7_{-0.4}^{+0.3}$ & $0.4_{-0.2}^{+0.2}$ & $0.4_{-0.3}^{+0.3}$ & $0.4_{-0.2}^{+0.4}$ & A2744 & Kawamata et al. (2015) \\
\hline HFF1P-i16 & 6.39 & $8.6_{-0.4}^{+0.3}$ & $0.3_{-0.2}^{+0.3}$ & $0.4_{-0.3}^{+0.3}$ & $0.3_{-0.2}^{+0.5}$ & A2744 & Kawamata et al. (2015) \\
\hline HFF1P-Y1 & 7.53 & $9.0_{-0.3}^{+0.2}$ & $0.9_{-0.2}^{+0.2}$ & $0.3_{-0.2}^{+0.2}$ & $0.3_{-0.2}^{+0.4}$ & A2744 & Kawamata et al. (2015) \\
\hline HFF1P-Y2 & 7.63 & $9.0_{-0.4}^{+0.3}$ & $0.8_{-0.2}^{+0.3}$ & $0.3_{-0.2}^{+0.2}$ & $0.5_{-0.3}^{+0.5}$ & A2744 & Kawamata et al. (2015) \\
\hline 8958 & 9.88 & $8.4_{-1.0}^{+0.9}$ & $-0.9_{-0.3}^{+0.2}$ & $0.4_{-0.1}^{+0.1}$ & $1.0_{-0.5}^{+0.8}$ & M0416 & Infante et al. (2015) \\
\hline 1859 & 9.46 & $8.3_{-0.2}^{+0.2}$ & $0.3_{-0.4}^{+0.3}$ & $0.2_{-0.2}^{+0.2}$ & $0.5_{-0.3}^{+0.5}$ & M0416 & Infante et al. (2015) \\
\hline 8364 & 9.17 & $9.1_{-0.1}^{+0.1}$ & $1.0_{-0.1}^{+0.1}$ & $0.2_{-0.2}^{+0.2}$ & $0.4_{-0.2}^{+0.4}$ & M0416 & Infante et al. (2015) \\
\hline 491 & 8.42 & $8.9_{-0.1}^{+0.1}$ & $1.0_{-0.1}^{+0.1}$ & $0.2_{-0.1}^{+0.2}$ & $0.2_{-0.1}^{+0.1}$ & M0416 & Infante et al. (2015) \\
\hline 8428 & 8.29 & $11.0_{-0.2}^{+0.2}$ & $1.7_{-0.2}^{+0.2}$ & $0.6_{-0.1}^{+0.1}$ & $2.5_{-0.1}^{+0.1}$ & M0416 & Infante et al. (2015) \\
\hline 1213 & 8.27 & $8.3_{-0.1}^{+0.1}$ & $0.2_{-0.1}^{+0.1}$ & $0.3_{-0.2}^{+0.2}$ & $0.4_{-0.2}^{+0.5}$ & M0416 & Infante et al. (2015) \\
\hline 4008 & 7.71 & $8.3_{-0.1}^{+0.1}$ & $0.2_{-0.2}^{+0.1}$ & $0.3_{-0.2}^{+0.2}$ & $0.2_{-0.1}^{+0.3}$ & M0416 & Infante et al. (2015) \\
\hline 3687 & 9.36 & $8.5_{-0.4}^{+0.4}$ & $0.5_{-0.3}^{+0.4}$ & $0.2_{-0.2}^{+0.2}$ & $0.6_{-0.3}^{+0.6}$ & M0416 & Infante et al. (2015) \\
\hline 4177 & 9.34 & $8.7_{-0.3}^{+0.2}$ & $0.7_{-0.1}^{+0.2}$ & $0.2_{-0.1}^{+0.2}$ & $0.2_{-0.1}^{+0.2}$ & M0416 & Infante et al. (2015) \\
\hline 3076 & 9.13 & $7.6_{-0.1}^{+0.1}$ & $0.5_{-0.1}^{+0.7}$ & $0.1_{-0.1}^{+0.1}$ & $0.1_{-0.1}^{+0.1}$ & M0416 & Infante et al. (2015) \\
\hline 5296 & 8.28 & $11.0_{-0.1}^{+0.1}$ & $2.6_{-0.4}^{+0.1}$ & $0.4_{-0.2}^{+0.2}$ & $1.2_{-0.2}^{+0.5}$ & M0416 & Infante et al. (2015) \\
\hline 1301 & 8.35 & $8.1_{-0.3}^{+0.2}$ & $0.2_{-0.1}^{+0.1}$ & $0.2_{-0.1}^{+0.2}$ & $0.1_{-0.1}^{+0.1}$ & M0416 & Infante et al. (2015) \\
\hline 3814 & 7.88 & $8.8_{-0.4}^{+0.4}$ & $0.6_{-0.2}^{+0.3}$ & $0.3_{-0.2}^{+0.2}$ & $0.7_{-0.3}^{+0.5}$ & M0416 & Infante et al. (2015) \\
\hline 1241 & 7.90 & $8.3_{-0.3}^{+0.2}$ & $0.2_{-0.1}^{+0.1}$ & $0.3_{-0.2}^{+0.2}$ & $0.1_{-0.1}^{+0.1}$ & M0416 & Infante et al. (2015) \\
\hline 3790 & 7.79 & $8.6_{-0.2}^{+0.2}$ & $0.8_{-0.1}^{+0.1}$ & $0.1_{-0.1}^{+0.2}$ & $0.1_{-0.1}^{+0.1}$ & M0416 & Infante et al. (2015) \\
\hline 4125 & 7.40 & $7.3_{-0.1}^{+0.1}$ & $0.8_{-0.1}^{+0.1}$ & $0.1_{-0.1}^{+0.1}$ & $0.1_{-0.1}^{+0.1}$ & M0416 & Infante et al. (2015) \\
\hline 6999 & 7.54 & $8.6_{-0.3}^{+0.2}$ & $0.5_{-0.1}^{+0.1}$ & $0.3_{-0.2}^{+0.2}$ & $0.1_{-0.1}^{+0.1}$ & M0416 & Infante et al. (2015) \\
\hline 7361 & 7.52 & $7.4_{-0.1}^{+0.1}$ & $0.3_{-0.1}^{+0.4}$ & $0.1_{-0.1}^{+0.1}$ & $0.1_{-0.1}^{+0.1}$ & M0416 & Infante et al. (2015) \\
\hline
\end{tabular}


Table 7

(Continued)

\begin{tabular}{lccrcrrr}
\hline \hline Galaxy ID & Redshift & $\begin{array}{c}\log M_{*} \\
\left(M_{\odot}\right)\end{array}$ & $\begin{array}{c}\log \text { SFR } \\
\left(M_{\odot} \mathrm{yr}^{-1}\right)\end{array}$ & $\begin{array}{c}\text { AGE } \\
(\mathrm{Gyr})\end{array}$ & $A_{v}$ & Cluster & References \\
\hline 1331 & 7.25 & $7.8_{-0.2}^{+0.2}$ & $0.6_{-0.1}^{+0.4}$ & $0.1_{-0.1}^{+0.1}$ & $0.1_{-0.1}^{+0.1}$ & M0416 & Infante et al. (2015) \\
1386 & 7.24 & $8.2_{-0.3}^{+0.2}$ & $0.3_{-0.1}^{+0.1}$ & $0.2_{-0.1}^{+0.3}$ & $0.1_{-0.1}^{+0.1}$ & M0416 & Infante et al. (2015) \\
146 & 7.25 & $8.3_{-0.3}^{+0.2}$ & $0.3_{-0.1}^{+0.1}$ & $0.2_{-0.1}^{+0.2}$ & $0.1_{-0.1}^{+0.1}$ & M0416 & Infante et al. (2015) \\
1513 & 6.66 & $10.2_{-0.1}^{+0.1}$ & $-2.1_{-2.6}^{+2.1}$ & $0.8_{-0.2}^{+0.1}$ & $1.2_{-0.2}^{+0.2}$ & M0416 & Infante et al. (2015) \\
\hline
\end{tabular}

Note. Same as in Table 6.

Lake et al. 2013; Schaerer et al. 2013; de Barros et al. 2014). To add robust constraints on the evolution of the SFR as a function of stellar mass, we only used objects detected in at least one of the IRAC images in our analysis. Among all the $z>6$ objects selected in FFs data set, only 18 are detected at 3.6 and/or $4.5 \mu \mathrm{m}$. Figure 6 shows the distribution of these candidates in the $\left(M^{\star}\right.$, SFR) plane, along with several $z \sim 7$ objects previously analyzed in Labbé et al. (2010) and McLure et al. (2011). As expected, the luminosity range covered by the FF data set is larger than previous surveys allowing to add more constraints at lower stellar mass. We used a $\chi^{2}$ minimization method to fit the evolution of the two properties and found an evolution at $z \sim 7$ given by

$$
\log [\mathrm{SFR}]=(0.88 \pm 0.44) \log \left[M^{\star}\right]-(6.97 \pm 3.95)
$$

where error bars represent the $1 \sigma$ confidence interval. This evolution is consistent with the trend observed by Labbé et al. (2010). As already demonstrated in Tasca et al. (2015), the relation between SFR and stellar mass currently observed over a large range of redshift seems higher than previous estimates of the main sequence by Elbaz et al. (2007) (cf. Figure 6). However, in our selection criteria we requested to have a detection in at least two NIR filters (UV rest-frame) that could bias our sample by selecting sources with the highest SFR in this redshift range. Therefore, the trend observed in this FF sample should be considered as an upper limit of the evolution.

The large sample of $z \sim 7$ and eight candidates allows us to study the evolution of the UV slope (hereafter $\beta$ slope), and therefore the reddening, as a function of stellar mass. We estimated the $\beta$ slope following Equation (1) of Dunlop et al. (2013) and the corresponding error bars were estimated from photometric errors. Figure 7 displays the evolution of the UV slope as a function of luminosity for all $z \sim 7$ candidates selected in the FF survey. The evolution is compatible with previous findings published in Wilkins et al. (2011) and Bouwens et al. (2012, 2015b). We also plot the evolution of $\beta$ as a function of the stellar mass, but only for candidates detected in at least one IRAC band. We compared this evolution with results published in Finkelstein et al. (2012) and found a similar evolution. We also studied the relationship between the star formation rate and galaxy mass in $z \sim 7$ candidates detected in at least one IRAC band. This sample seems to follow the trend observed for $z \sim 2$ galaxies (Daddi et al. 2007; Elbaz et al. 2007). A similar trend has been deduced from the analysis of $\approx 1700$ LBGs at $z \sim 3-6$ (Schaerer et al. 2013; de Barros et al. 2014) with a better stellar mass coverage. From our sample of IRAC detected $z \sim 7$ galaxies, we confirm that massive galaxies seem more affected by dust attenuation than smaller galaxies. To test this result at such high redshift, we need to strongly increase the number of $z \geqslant 7$ candidates bright enough to be detected in IRAC data.

The star formation history in very high redshift galaxies can be studied through the specific SFR (sSFR), the ratio between the SFR, and the stellar mass of a given galaxy. We used the sample of $z \sim 7$ and eight FF candidates to estimate the sSFR at such high redshift. As before, we only used galaxy candidates that are detected in at least one IRAC band to have a more robust estimate of their stellar mass. To study the evolution of this quantity as a function of redshift, we only considered objects that have a stellar mass within the interval 2.5-7.5 $\times 10^{9} M_{\odot}$. Errors bars were obtained by quadratically adding the errors on the SFR and the stellar mass (Figure 6). We modified the parameterization found from the VUDS survey (Tasca et al. 2015) for galaxies at $z>2.4$ as

$$
\mathrm{sSFR}=0.2 \times(1+z)^{1.2} .
$$

Our values are in perfect agreement with previous findings at lower redshift (Stark et al. 2013; González et al. 2014 and Tasca et al. 2015).

\subsection{Size}

The size of our objects was computed using the SExtractor FLUX_RADIUS and setting the flux fraction parameter to 0.5 to get the half-light radius. We corrected the size for PSF broadening following the method described in Oesch et al. (2010c): $r=\sqrt{r_{\mathrm{SEx}}^{2}-r_{\mathrm{psf}}^{2}}$, where $r_{\mathrm{SEx}}$ is the half-light radius and $r_{\mathrm{psf}}$ the PSF of the F140W image. We also took into account the amplification of the light by the cluster making the observed size larger. We used the scale factor between the size on the sky and the physical size computed from Wright (2006). Recent studies benefited from HST image quality to study the evolution of the size of $z \sim 8$ objects selected in FF data sets as a function of the UV luminosity (e.g., Kawamata et al. 2015; Laporte et al. 2014, 2015). Figure 9 displays this evolution and shows that our $z \sim 8$ objects are consistent with the trend observed by previous authors.

We applied the same method to compute the size of $z \sim 6-7$ objects in our sample and in those published in Infante et al. (2015). We also used results from Kawamata et al. (2015) to study the size-luminosity relation at this redshift range as seen by the three first FF data sets. We took advantage of the large number of $z \sim 6-7$ candidates already selected in the FF data (cluster and parallel fields) to compute an average evolution (Figure 10). We used Equation (4) from Ono et al. (2013) to constrain the SFR densities for these objects, but we failed to obtain strong constraints due to the large uncertainties of radius which can only be reduced by further increasing the number of $z \sim 6-7$ objects. Nevertheless, The distribution of the FFselected candidates in the $\left(r, M_{1500}\right)$ plane is consistent with 


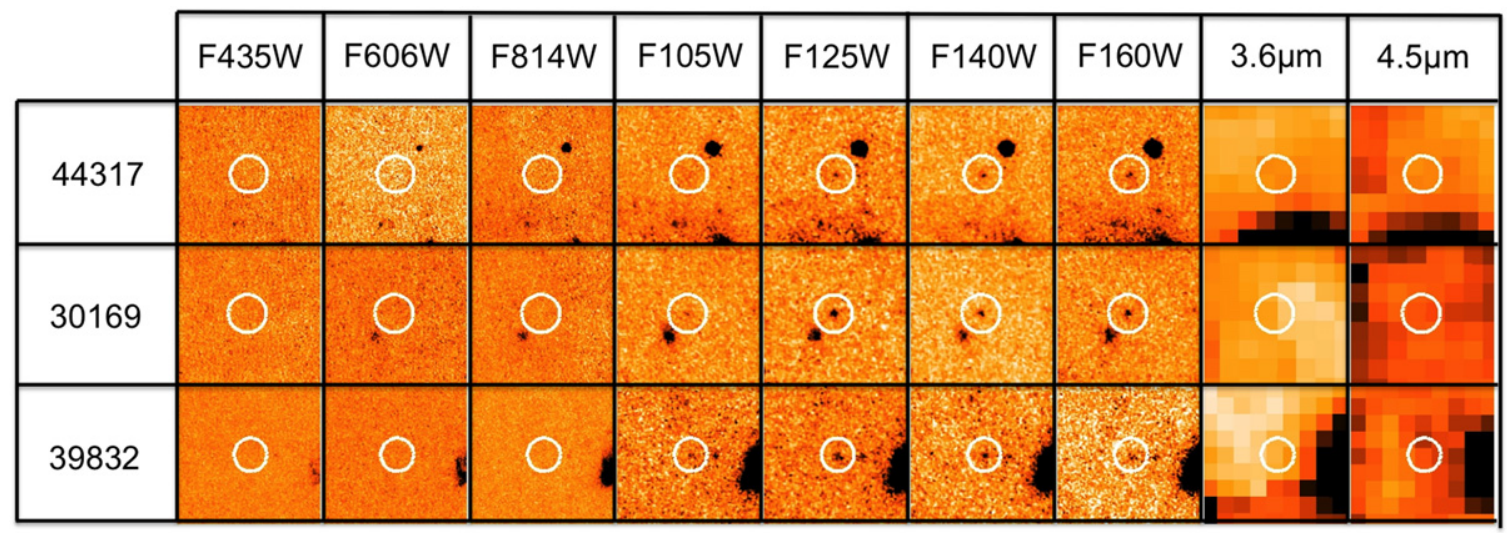

Figure 3. Thumbnail images of $z \sim 8$ candidates selected on the parallel field. Each stamp is $5^{\prime \prime} \times 5^{\prime \prime}$ and the position of each candidate is displayed as a white 0 ." 6 radius circle.

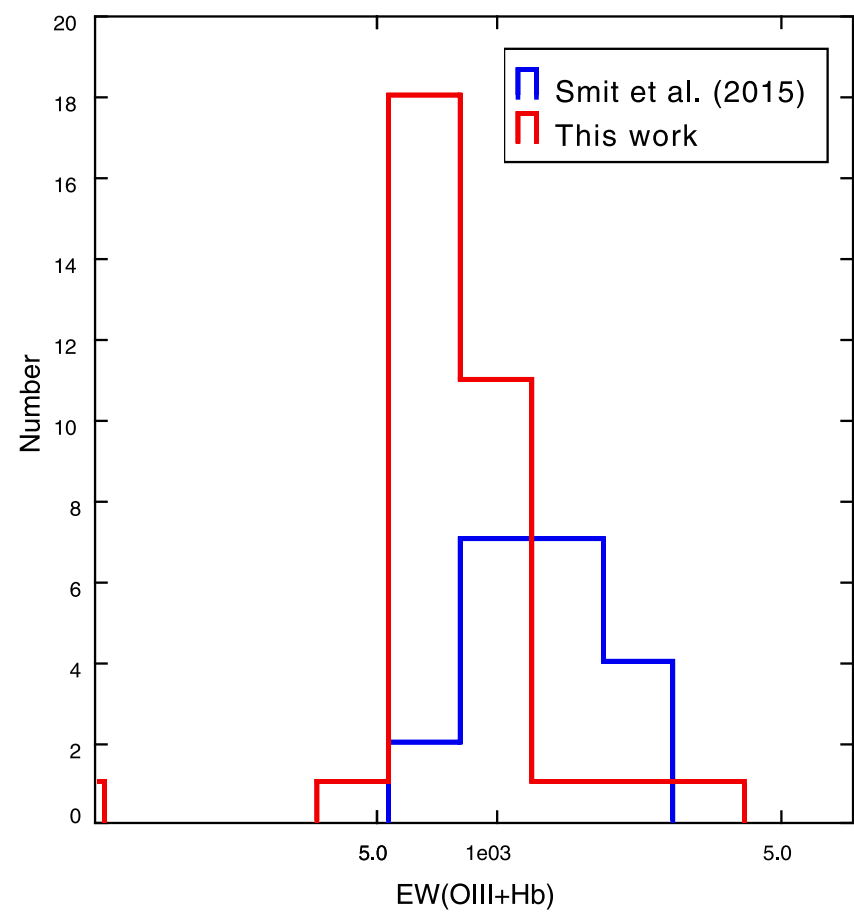

Figure 4. Distribution of the estimated equivalent widths of $[\mathrm{O} \mathrm{III}]+\mathrm{H} \beta$ for $z \sim 7$ objects selected in the three first Frontier Fields data set (red) compared with the distribution for 20 Lyman break galaxies (blue) discussed in Smit et al. (2015).

previous results published at $z \sim 7$ (Oesch et al. 2010a; CurtisLake et al. 2016 and Holwerda et al. 2015).

Recently, Curtis-Lake et al. (2016) claimed no evolution in the size of Lyman break galaxies with redshift. We tried to investigate this conclusion using our sample of FF-selected objects at $z \sim 7$ and 8 . The average size of $(0.3-1) L_{z=3}^{\star}$ galaxies at $z \sim 7$ is $r_{z=7}(\mathrm{kpc})=0.80 \pm 0.18$, similar to the value computed from HUDF objects (Oesch et al. 2010a). In the same way, we estimated the mean size for $(0.3-1) L_{z=3}^{\star}$ objects at $z \sim 8$ as $r_{z=8}(\mathrm{kpc})=0.45 \pm 0.15$, which is also consistent with previous results. Therefore, we cannot exclude evolution in the size of Lyman break galaxies between $z \sim 7$ and 8 , although the number of $z \sim 8$ candidates selected in the three first FF clusters is still insufficient to draw any firm conclusions.

\section{DISCUSSION}

\subsection{Comparison of Our Sample with Previous Studies}

MACS J0717.5+3745 is part of the CLASH survey, and several searches for high- $z$ objects have been done using shallower HST data. As shown by Bouwens et al. (2014) and Bradley et al. (2014), no F140W $<27.5 z \sim 9$ and 10 candidates has been selected behind this lensing cluster. We confirm this result and push the limits deeper by one magnitude in F140W.

We used deeper ACS data to check the non-detection of previous high- $z$ candidates. Bradley et al. (2014) published 15 candidates with photometric redshifts $>5.5$. One object, namely MACS 0717-0247, is clearly detected in all ACS FF images and thus is no longer a good high- $z$ candidate and MACS 0717-0844 is detected in $\mathrm{F} 606 \mathrm{~W}$, explaining why it is not in our sample. Moreover, among the 15 objects, three are out of the field of view covered by the FF images (MACS 0717-0145, MACS 0717-0166, and MACS 0717-0390). We recovered MACS 0717-0234 (\#46206), MACS 0717-0859 (\#33447, confirmed at $z=6.39$ by spectroscopy in Vanzella et al. 2014), MACS 0717-1077 (\#30458), MACS 0717-1730 (\#21962, confirmed at $z=6.39$ by spectroscopy in Vanzella et al. 2014), and MACS 0717-1991 (\#15440). Therefore, all the good $z>6$ objects published in Bradley et al. (2014) located in the FF area are also in our selection; the difference between the two samples comes from the depth difference between the CLASH and FF data sets, thus we only added fainter candidates and excluded the CLASH candidates that were detected in the deep ACS taken for FF.

The size of our sample of $z \sim 6-7$ candidates is comparable to those built using previous FF data (e.g., Atek et al. 2015b; Kawamata et al. 2015). However, the number of $z \geqslant 8$ objects in the cluster field strongly differs from what has been found by previous authors (McLeod et al. 2015; Infante et al. 2015; Laporte et al. 2015), suggesting either a strong influence of cosmic variance $(\mathrm{CV})$ or that our selection criteria are not wellsuited for selecting very high- $z$ objects (see Section 6.5).

We also used the preliminary results of the GLASS survey (Schmidt et al. 2014) around MACS 0717. In that paper, the authors combined three different selection methods to reduce the incompleteness of their sample and retained 21 objects using CLASH data on that cluster (Postman et al. 2012). They added the 15 objects selected by Bradley et al. (2014) to their samples already discussed above. For the six remaining objects, three are out of the field of view covered by FF images, two are 


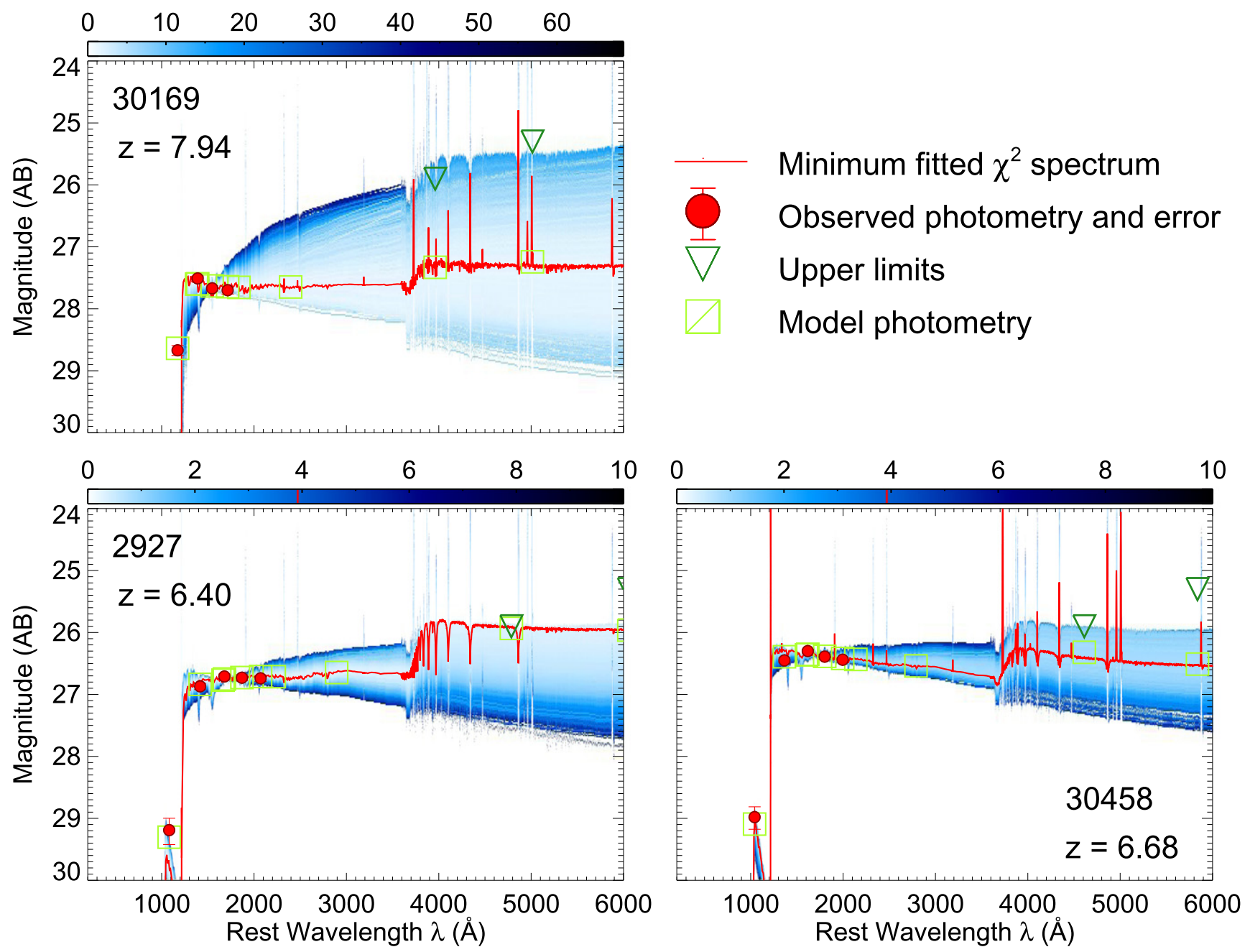

Figure 5. Example of SED-fitting results using iSEDfit (Moustakas et al. 2013) for three objects among our MACS 0717 sample (cluster and parallel fields). Nondetections are plotted at $3 \sigma$ with green triangles, the red lines display the best fit, the green squares are photometric magnitudes of the best fit and the blue region shows several of the models we used to fit the SED. The color bar is a $\chi^{2}$ scale, indicating the quality of the fit.

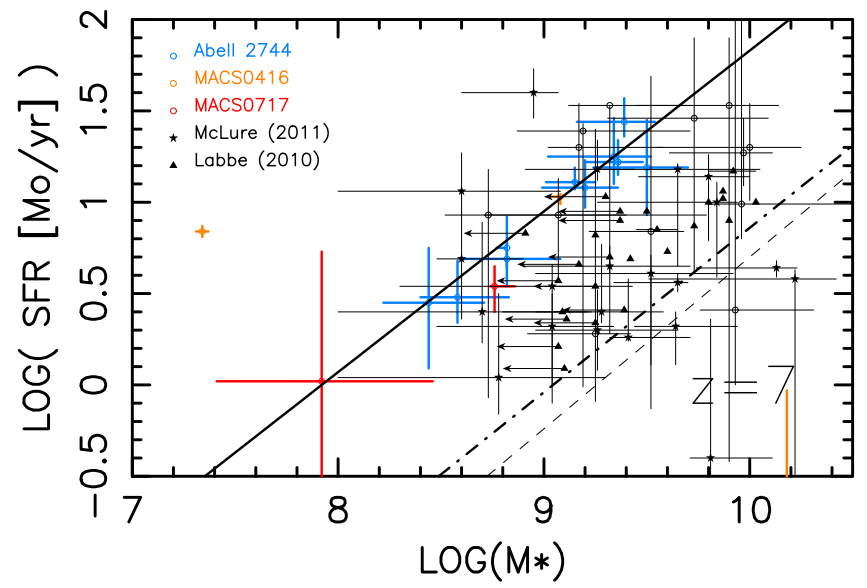

Figure 6. Evolution of the SFR as a function of the stellar mass for all of the $z \sim 7$ candidates selected in the three first Frontier Fields data set (Abell 2744 in blue, MACS 0416 in gray, and MACS 0717 in red). We overplotted objects (black points) analyzed in Labbé et al. (2010) and McLure et al. (2011). The solid line shows the best fit of the SFR- $M^{\star}$ relation deduced by $\chi^{2}$ minimization using Frontier Fields selected candidates. The dashed line displays the parameterization deduced from SDSS galaxy at $z \sim 1$ (Elbaz et al. 2007) and the dotted-dashed line shows the relation published by Labbé et al. (2010). clearly detected on F606W and could not be at such high redshift (\#1492 and \#1656), and one object does not fulfill the color criteria we requested (\#1841). We compared our $z \geqslant 7$ selected candidates with samples recently published in Schmidt et al. (2016) and noticed that the only "good" dropout they selected in MACS 0717 (MACS 0717-00908) is clearly detected in all ACS images, suggesting a low- $z$ solution for that object and explaining why it is not in our sample. Moreover, they detected emission line for three $z \sim 7$ candidates in MACS 0717 field: two have already been discussed in Vanzella et al. (2014) and MACS 0717-00370 displays a line at a signal-to-noise ratio of $\sim 3$ $\left(f_{\mathrm{Ly} \alpha}=1.9 \times 10^{-17} \mathrm{erg} \mathrm{s}^{-1} \mathrm{~cm}^{-2}\right)$. This last source is also included in our $z \geqslant 6$ sample (\#13963) with a photometric redshift ranging from 6.3 to 6.8 (a $1 \sigma$ confidence interval, see hereafter for details). Assuming that the detected emission line is $\operatorname{Ly} \alpha$, this would place this object at $z=6.51$. For future spectroscopic follow-up, it is interesting to note that for the remaining objects no emission line was detected for all of these objects within the framework of the GLASS survey, pushing the flux limit for Ly $\alpha$ down to $1.0 \times 10^{-17} \mathrm{erg} \mathrm{s}^{-1} \mathrm{~cm}^{-2}$ at $2 \sigma$. 
Table 8

Number Densities of $z>6.5$ Objects Computed using Half of the Frontier Fields Survey

\begin{tabular}{|c|c|c|c|c|c|c|c|c|}
\hline$\langle z\rangle$ & $M_{1500}$ & $\begin{array}{c}\Phi\left(M_{1500}\right) \\
\left(\times 10^{-4} \mathrm{Mpc}^{-3}\right)\end{array}$ & $\langle z\rangle$ & $M_{1500}$ & $\begin{array}{c}\Phi\left(M_{1500}\right) \\
\left(\times 10^{-4} \mathrm{Mpc}^{-3}\right)\end{array}$ & $\langle z\rangle$ & $M_{1500}$ & $\begin{array}{c}\Phi\left(M_{1500}\right) \\
\left(\times 10^{-4} \mathrm{Mpc}^{-3}\right)\end{array}$ \\
\hline 7 & $\begin{array}{l}-21.75 \pm 0.50 \\
-20.75 \pm 0.50 \\
-20.00 \pm 0.25 \\
-19.50 \pm 0.25 \\
-19.00 \pm 0.25 \\
-18.50 \pm 0.25 \\
-18.00 \pm 0.25 \\
-17.25 \pm 0.50\end{array}$ & $\begin{array}{c}<0.27 \\
0.64 \pm 0.53 \\
2.85 \pm 1.40 \\
4.21 \pm 1.85 \\
7.06 \pm 2.38 \\
13.7 \pm 7.56 \\
22.7 \pm 12.4 \\
47.4 \pm 41.8\end{array}$ & 8 & $\begin{array}{l}-21.25 \pm 0.50 \\
-20.50 \pm 0.25 \\
-19.75 \pm 0.50 \\
-18.75 \pm 0.50 \\
-17.75 \pm 0.50 \\
-16.75 \pm 0.50\end{array}$ & $\begin{array}{c}<0.30 \\
1.09 \pm 0.70 \\
2.58 \pm 1.37 \\
7.25 \pm 4.04 \\
14.7 \pm 13.9 \\
67.7 \pm 65.9\end{array}$ & 9 & $\begin{array}{l}-20.75 \pm 0.50 \\
-19.75 \pm 0.50 \\
-18.75 \pm 0.50 \\
-17.75 \pm 0.50\end{array}$ & $\begin{array}{c}<0.33 \\
0.57 \pm 0.46 \\
2.27 \pm 1.82 \\
16.6 \pm 13.3\end{array}$ \\
\hline
\end{tabular}

Table 9

Parameterization of the UV Luminosity Function

\begin{tabular}{|c|c|c|c|c|}
\hline$<z>$ & References & $M^{\star}$ & $\Phi^{\star}$ & $\alpha$ \\
\hline \multirow[b]{3}{*}{7} & This work & $-20.33_{-0.47}^{+0.37}$ & $0.37_{-0.11}^{+0.12} \times 10^{-3}$ & $-1.91_{-0.27}^{+0.26}$ \\
\hline & Bouwens et al. (2015b) & $-20.87 \pm 0.26$ & $0.29_{-0.12}^{+0.21} \times 10^{-3}$ & $-2.06 \pm 0.13$ \\
\hline & McLure et al. (2013) & $-19.90_{-0.28}^{+0.23}$ & $1.09_{-0.45}^{+0.56} \times 10^{-3}$ & $-1.90_{-0.23}^{+0.22}$ \\
\hline \multirow{2}{*}{8} & This work & $-20.32_{-0.26}^{+0.49}$ & $0.30_{-0.19}^{+0.85} \times 10^{-3}$ & $-1.95_{-0.40}^{+0.43}$ \\
\hline & Bouwens et al. (2015b) & $-20.63 \pm 0.36$ & $0.21_{-0.11}^{+0.23} \times 10^{-3}$ & $-2.02 \pm 0.23$ \\
\hline \multirow{3}{*}{9} & This work & -20.45 (fixed) & $0.70_{-0.30}^{+0.30} \times 10^{-4}$ & $-2.17_{-0.43}^{+0.41}$ \\
\hline & McLeod et al. (2015) & -20.1 & $2.51_{-1.39}^{+1.46} \times 10^{-4}$ & -2.02 (fixed) \\
\hline & Bouwens et al. (2015c) & -20.45 & $1.0 \times 10^{-4}$ & -2.3 \\
\hline
\end{tabular}
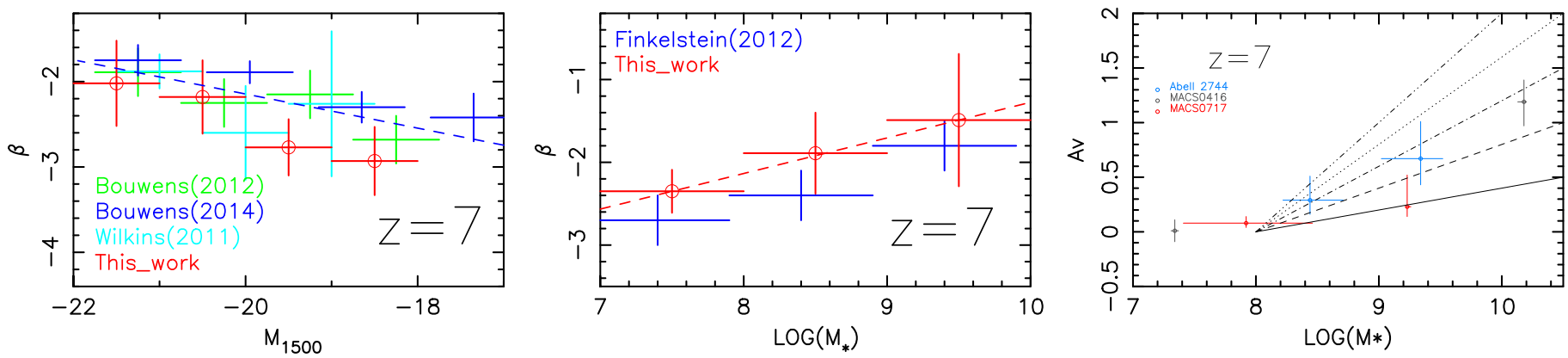

Figure 7. Left: evolution of the UV slope as a function of the UV luminosity deduced from all $z \sim 7$ candidates selected in the first three Frontier Fields (red points) compared with previous findings (Wilkins et al. 2011; Bouwens et al. 2012, 2015b). The dashed line shows the evolution computed by Bouwens et al. (2015b) from a sample of $\approx 200$ galaxies. Middle: evolution of the UV slope as a function of the stellar mass computed from objects detected in at least one IRAC band (red points) compared with the evolution found in Finkelstein et al. (2012). Right: evolution of the reddening as a function of galaxy mass for all the candidates selected in the three first Frontier Fields (Abell 2744 in blue, MACS 0416 in gray, and MACS 0717 in red). We also plot the trend observed by Schaerer \& de Barros (2010) $\left(A_{v}=\log \left(\frac{M_{\star}}{10^{8} M_{\odot}}\right)^{n}\right)$, assuming several values of $n(0.2$, solid line, 0.4 , dashed line, 0.6, dotted-dashed line, 0.8 , dotted line, and 1.0 the triple dotted-dashed line).

\subsection{Contamination of the Samples}

Among all the possible sources of contaminants in a high- $z$ samples, the most likely are the low-mass stars, the transient objects, or the SNe or the low-z interlopers. In the following section we discuss the contamination rate of our sample by several types of sources.

Low-mass stars have colors that could enter our selection criteria, but they should be unresolved on single-epoch HST data. We computed expected colors for low-mass stars from a set of 225 stellar templates of M, L, and T dwarfs (Burgasser et al. 2004, 2006a, 2006b, 2008; Cruz et al. 2004; Burgasser \&
McElwain 2006; Chiu et al. 2006; McElwain \& Burgasser 2006; Reid et al. 2006; Burgasser 2007; Liebert \& Burgasser 2007; Looper et al. 2007; Siegler et al. 2007; Sheppard \& Cushing 2009; Kirkpatrick et al. 2010). As shown in Figure 1, the selection windows we defined to select $z \gtrsim 6$ objects exclude the large part of low-mass star colors. However, we noted that $34 \%$ of M, L, and T dwarfs we simulated have colors consistent with $z \sim 6$ objects, but only $2 \%$ of these stars have colors that fulfill the criteria defined for $z \sim 8$ objects.

However, to remove the stellar hypothesis for all our candidates, we first check the SExtractor stellarity parameters and then measure their sizes on the HST images using the 


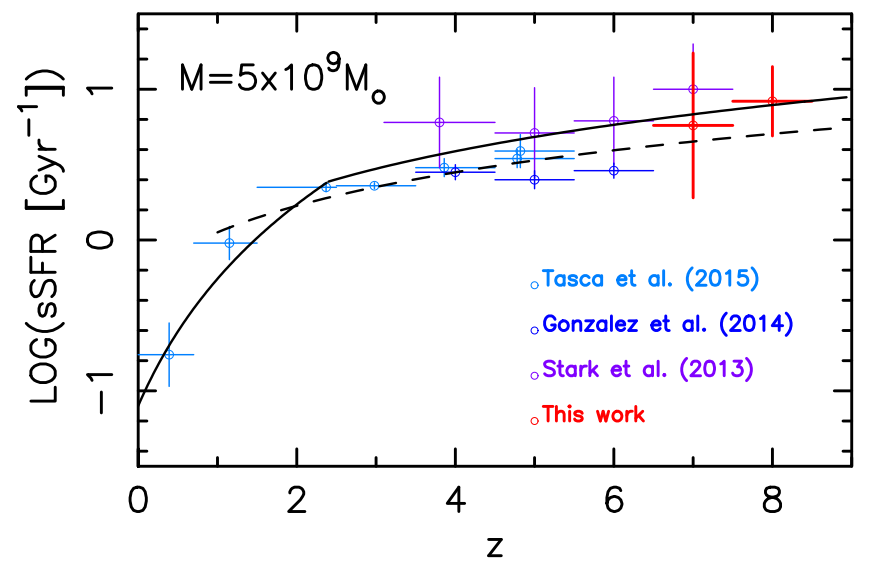

Figure 8. Specific star formation rate (sSFR) as a function of redshift for galaxies with stellar mass as of $M^{\star} \sim 5 \times 10^{9} M_{\odot}$. We compare the sSFR we deduce from Frontier Field candidates detected in at least one IRAC band with results published in Tasca et al. (2015), González et al. (2014) and Stark et al. (2013). The solid line shows an updated version of the parameterization discussed in Tasca et al. (2015) and the dashed line displays the evolution found by González et al. (2014).

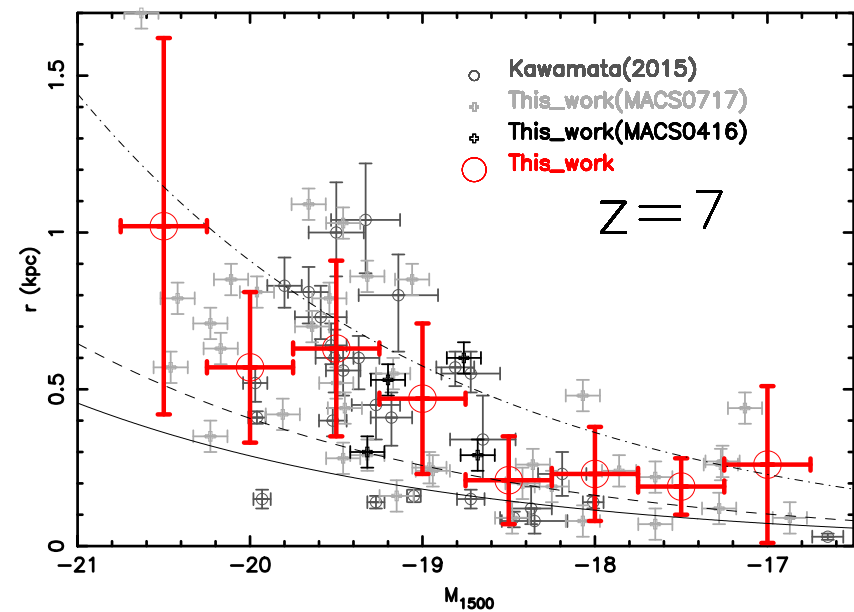

Figure 9. Evolution of the size of $z \sim 6-7$ candidates selected behind the 3 first Frontier Fields as a function of the UV Luminosity: Abell 2744 (Kawamata et al. 2015), MACS 0416 (Laporte et al. 2015 and this work based on Infante et al. 2015 samples) and MACS 0717 (this paper). The red points show the average radius per bin of $0.5 M_{1500}$, error bars are the standard deviation. We overplotted several size-luminosity relation using different assumptions on the SFR densities (10-solid line, 5-dashed line, 1-dotted dashed line, $M_{\odot} \mathrm{yr}^{-1} \mathrm{kpc}^{2}$ ).

SExtractor half-light radius for each object. For the cluster sample, excluding two objects that display a stellarity of 0.4 (\#25990 and \#46005), all of the candidates have a CLASS_STAR parameter $<0.1$, meaning that all of our candidates have a morphology inconsistent with a star. Moreover, all our objects are resolved on the F160W image, making the star hypothesis unlikely. The same conclusion could be made for the parallel field with all objects have a stellarity parameter $<0.1$. We measured the size of our objects in Section 5.4 and showed that five objects among the two samples are unresolved on the HST images: 1 $z \sim 8$ candidate (\#39832) and $4 z \sim 7$ objects (\#3119, \#6576, \#66722, and \#91692).

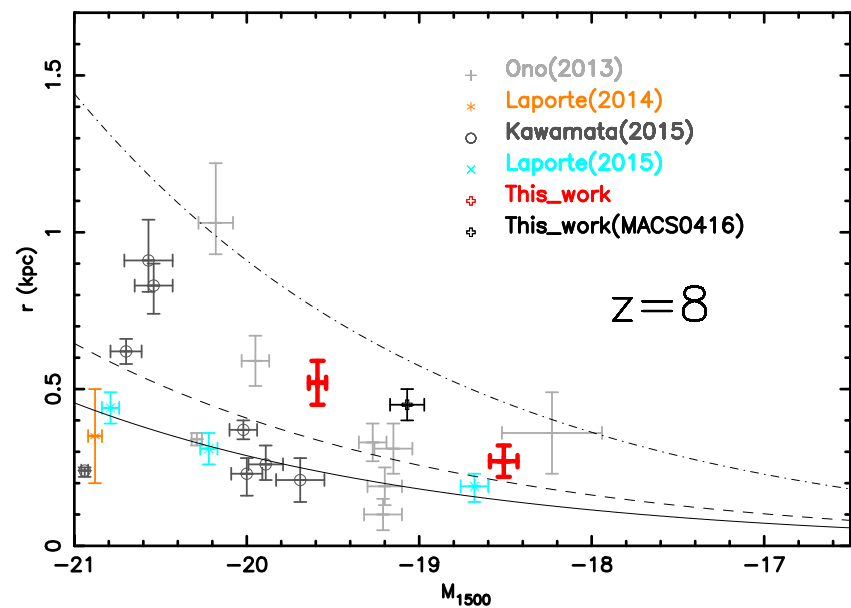

Figure 10. Evolution of the size of $z \sim 8$ candidates selected behind the three first Frontier Fields as a function of the UV Luminosity: Abell 2744 (Laporte et al. 2014 and Kawamata et al. 2015), MACS 0416 (Infante et al. 2015, and Laporte et al. 2015) and MACS 0717 (this paper). We compared this evolution with results from the HUDF 2012 campaign (Ono et al. 2013). We overplotted several size-luminosity relation using different assumptions on the SFR densities (10-solid line-5—dashed line-1—dotted dashed line- $\left.M_{\odot} \mathrm{yr}^{-1} \mathrm{kpc}^{2}\right)$.

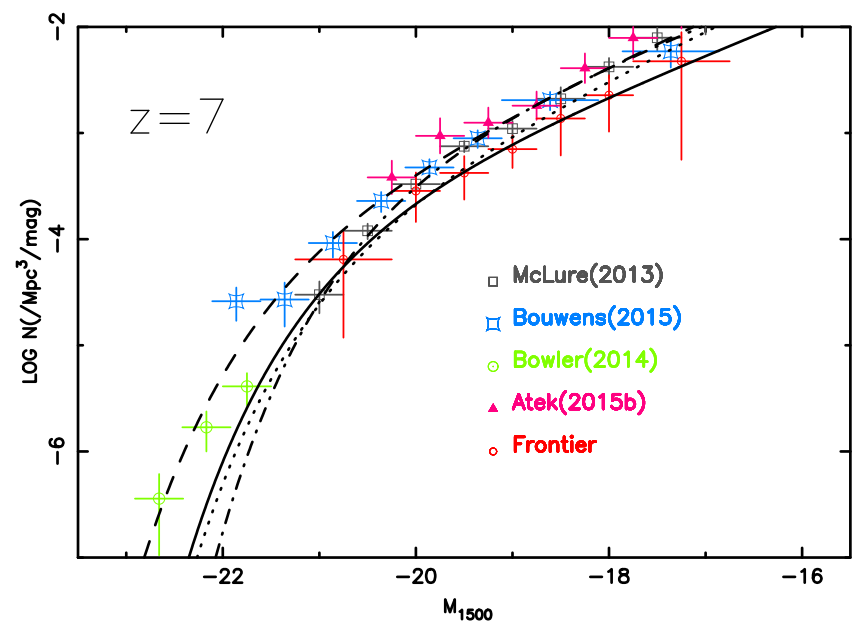

Figure 11. UV luminosity function at $z \sim 7$ computed using the first half of the Frontier Fields data. Number densities estimated from this study are in red. We overplotted results from other groups using other data sets (McLure et al. 2013; Bowler et al. 2014; Atek et al. 2015a; Bouwens et al. 2015b). The solid line displays the parameterization we deduced from this study, the dotted-dashed line shows the shape published by Bouwens et al. (2015b), the dashed line is from Bowler et al. (2014), and the dotted line is from McLure et al. (2013).

\subsection{Completeness of the Selection Method}

The method we used to extract and select the very high- $z$ candidates implies incompleteness. In other words, we are not selecting all $z \gtrsim 6$ objects that are effectively in our data set, thus we are missing some of them. One of the goals of a very high- $z$ study is to keep this incompleteness small and to take it into account in all statistical analyses.

In our case the incompleteness is due to the extraction method and the selection criteria described in Section 4. We applied the six-step correction procedure summarized below.

1. We estimated colors of $\approx 700,000 z \gtrsim 5$ galaxies from standard templates (see references in Section 5) with 


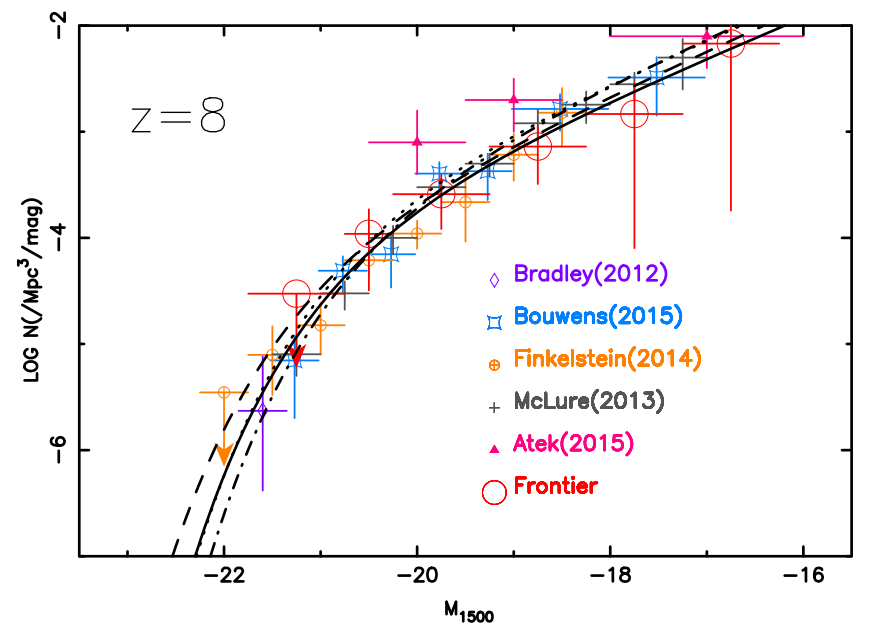

Figure 12. UV luminosity function at $z \sim 8$ computed using the first half of the Frontier Fields data. Number densities estimated from this study are in red. we overplotted results from other groups using other data sets (Bradley et al. 2012; McLure et al. 2013; Bouwens et al. 2015b). The solid line displays the parameterization we deduced from this study, the dotted-dashed line shows the shape published by Bouwens et al. (2015b), the dashed line is from Bradley et al. (2012), and the dotted line is from McLure et al. (2013).

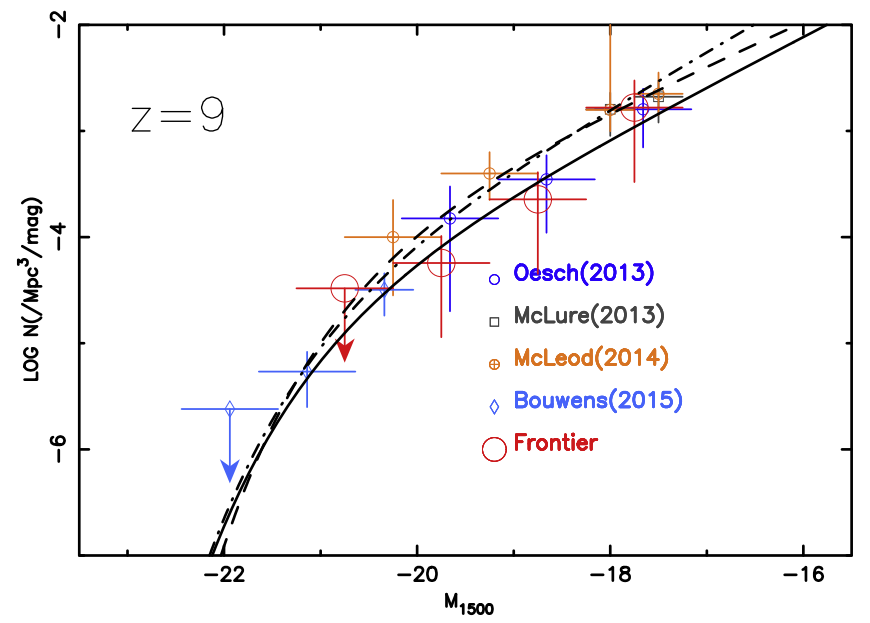

Figure 13. UV luminosity function at $z \sim 9$ computed using the first half of the Frontier Fields data. Number densities estimated from this study are in red. We overplotted results from other groups using other data sets (Lorenzoni et al. 2011; McLure et al. 2013; Oesch et al. 2013; McLeod et al. 2015 and Bouwens et al. 2015c). The solid line displays the parameterization we deduced from this study, the dotted-dashed line shows the shape published by McLure et al. (2013), and the dashed line is from Bouwens et al. (2015c).

magnitudes ranging from 22 to 32 in the filter following the position of the Lyman break and redshifts from 5 to 12.

2. We generated several lists of positions from an image constructed from the detection image where all objects have been masked.

3. For each object in our mock catalog, we associated a size assuming a log-normal distribution with a mean value of 0 ." 15 and a sigma of 0. .07 (Oesch et al. 2010a) in the source plane and lensed it through the cluster.

4. We then added all of these objects on the real images using the mkobjects routine of IRAF.

5. We applied the extraction method and selection criteria we used to select real objects.

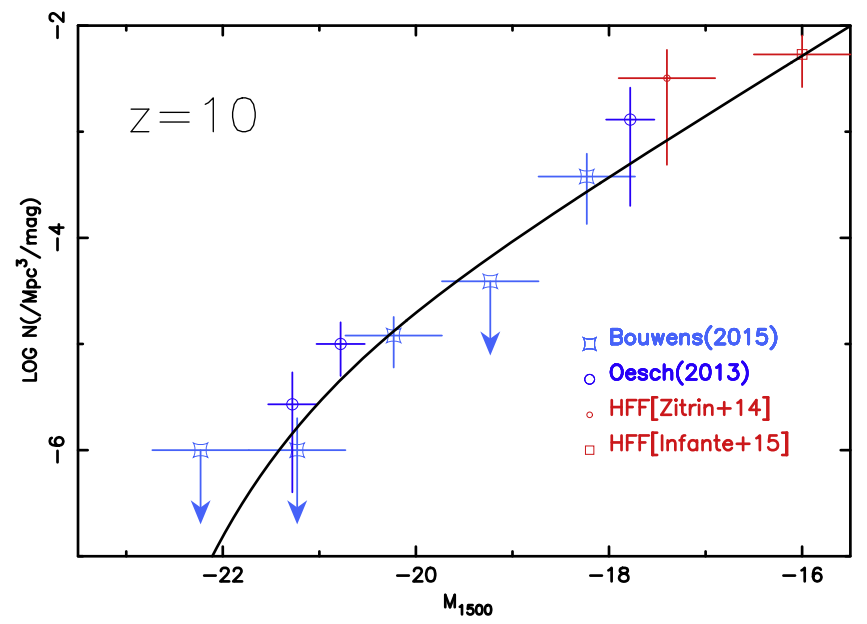

Figure 14. UV luminosity function at $z \sim 10$ computed using the first half of the Frontier Fields data. No $z \sim 10$ objects has been selected in the last Frontier Fields data set. We computed number densities based on previous $z \sim 10$ candidates selected on the two first Frontier Fields data set (and Infante et al. 2015). The solid line displays the parameterization published in Bouwens et al. (2015b).

6. Incompleteness levels were deduced by comparing the list of objects we selected at the end with the input list of mock objects.

The conclusion of this analysis is that we reach a $\sim 70 \%$ completeness at $m_{1500}^{\mathrm{RF}} \sim 29.5 A B$ for our two selection functions.

\subsection{Constraints on the $U V L F$}

One of the main goals of the FF legacy program is to constrain the evolution of the galaxies during the first billion years of the universe through the evolution of the UV LF, especially by adding robust constraints at faint luminosities. We deduced number densities of our photometric samples by taking into account the uncertainties on each redshift. Indeed, over the redshift interval covered by this survey, uncertainties on photometric redshifts are not negligible. We applied a standard Monte Carlo method based on the redshift probability distribution (e.g., Laporte et al. 2015) and summarized as follows.

1. At each iteration, we assign a photometric redshift based on the redshift probability distribution.

2. We compute the UV luminosity based on this redshift and the SED of each object.

3 . We repeat the previous steps $N$ times to obtain a sample with $N$ times the size of the original sample but with the same distribution in redshift.

4. We distribute objects into redshift and magnitude bins (e.g., $z \pm 0.5$ with $z=7-10$ ), divide the number of objects by the number of iterations $N$ and the volume explored estimated from the detection picture.

5. Error bars include statistical uncertainties and CV (Trenti \& Stiavelli 2008).

We deduced upper limits based on Poisson statistics. The resulting number densities are presented on Table 8 .

To study the evolution of the shape of the UV LF, we adopted the Schechter parameterization (Schechter 1976) and estimated the three parameters, so called $M^{\star}, \Phi^{\star}$, and $\alpha$, using a $\chi^{2}$ minimization method and previous published densities covering other luminosities ranges. Table 9 presents the parameterization 

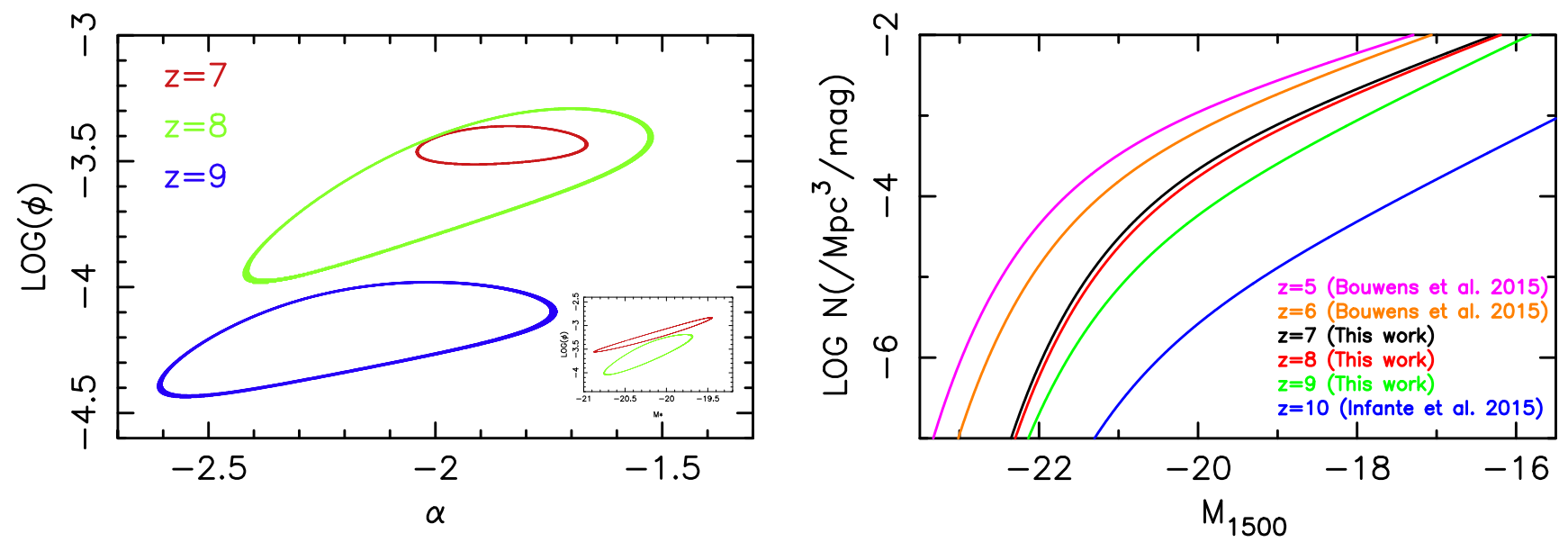

Figure 15. Left: $1 \sigma$ confidence intervals on the Schechter parameterization we deduced from number densities computed using all selected objects in the three first Frontier Fields. It shows a strong evolution between $z \sim 8$ and 9 of the $\Phi^{\star}$ parameter. The smaller panel shows the $1 \sigma$ confidence intervals for $M^{\star}$ and $\Phi^{\star}$ confirming an evolution of $\Phi^{\star}$ parameter. Right: evolution of the UV LF found in this study at $z \sim 7,8$, and 9 . For comparison purposes we overplotted the shape of the UV LF published in Bouwens et al. (2015b) at $z \sim 5$ and 6 and Infante et al. (2015) at $z \sim 10$.

for the redshift range covered in this study, and Figures 11-14 show the shape of the UV LF at $z \sim 7-10$, respectively. With half of the full FF data, we are probing the faint end of the UV LF up to the highest redshift and confirm the shape found by previous studies. However, it appears that the evolution between $z \sim 8$ and 9 is stronger than what has been previously observed (see Figure 15), suggesting a deficit of $z \sim 8.5$ objects (see Section 6.5). The evolution of the $1 \sigma$ confidence intervals from $z \sim 7$ to 9 shows a clear evolution in $\Phi^{\star}$ as already noticed by Bouwens et al. (2015b) with relatively small evolution in $\alpha$ (see Figure 15).

\subsection{A Deficit of $z>8.5$ Galaxies?}

The number of $z \geqslant 8$ objects selected behind MACS 0717 is lower than what was found behind the two first FF clusters. We computed the expected number of $z \sim 8$ galaxies detected at $5 \sigma$ in the MACS 0717 FF data, assuming the UV LF evolution published in Bouwens et al. (2015b) and the mass model provided by the CATS team. Taking into account the uncertainties on the LF parameters, the number of $z>7.5$ objects should be $2.98_{-1.14}^{+5.55}$, showing that at least one object should be detected on the FF images. However, the area effectively covered at very high- $z$ redshift by HST images is small enough to be strongly affected by CV. We used the method described in Trenti \& Stiavelli (2008) to account for $\mathrm{CV}$ in the expected number of objects. Based on the interval of $z>8$ objects detected at more than $5 \sigma$ in our data, the $\mathrm{CV}$ enlarges the range of expected objects to between 0 to 10.6 such that an absence of any $z>8$ candidates behind MACS 0717 cluster is possible.

\subsection{The Star Formation Rate Density}

One can constrain the role played by the first galaxies during the epoch of reionization by estimating the densities of UV photons they produced and how these densities evolve with redshift (e.g., Bouwens et al. 2015a). This quantity is related to the SFRd occurring as a function of redshift and is deduced from

$$
\rho_{\mathrm{SFR}}=1.25 \times 10^{-28} \int_{0.03 L_{z=3}^{\star}}^{\infty} \Phi\left(L_{1500}\right) d L_{1500}
$$

where $\Phi\left(L_{1500}\right)$ is the UV LF estimated in the previous section (e.g., Schiminovich et al. 2005). Thanks to the magnification applied by lensing clusters, we can integrate the UV LF down to $0.03 L_{z=3}^{\star}$ (i.e., $M_{1500} \sim-17$ ).

We corrected these densities for dust attenuation following the method described in Schiminovich et al. (2005) with the $\beta$ slopes published in Bouwens et al. (2012). To have a homogeneous determination of the star formation rate densities, we used previous UV LF parameterizations in several redshift intervals published in Wyder et al. (2005), van der Burg et al. (2010), McLure et al. (2009), Oesch et al. (2010b), Reddy \& Steidel (2009), Oesch et al. (2012), Bouwens et al. (2015b), and McLeod et al. (2015). We deduced $1 \sigma$ errors bars on each density based on uncertainties on the Schechter parameters. However, in cases where the parameters are fixed to a given value, we assumed uncertainties of $0.20 \%, 0.20 \%$, or $20 \%$ of the values, respectively, for $\alpha, M^{\star}$, and $\Phi^{\star}$.

The densities computed using half of the full FF data are in good agreement with previous results at $z<8$, and confirm the change of the slope in the evolution of the SFRd as a function of redshift beyond $z \sim 8$ (Bouwens et al. 2011a; Oesch et al. 2014; Ishigaki et al. 2015). The evolution of the SFRd as a function of redshift could be well-fitted by the equation given in Cole et al. (2001) up to $z \sim 8$ and given by

$$
\rho_{\mathrm{SFR}}(z)=\frac{a+b z}{1+\left(\frac{z}{c}\right)^{d}} h
$$

where we estimated $(a, b, c, d)=(0.0,0.05,2.55,3.30)$ using $\chi^{2}$ minimization.

However, the previous parameterization does not take into account the slope change at $z>8$ that is well-fitted by Equation (39) of Ishigaki et al. (2015) given by

$$
\rho_{\mathrm{SFR}}(z)=\frac{2 \rho_{\mathrm{UV}, z=8}}{10^{a(z-8)}+10^{b(z-8)}}
$$

where $(a, b)=(0.21,0.58)$ were estimated by $\chi^{2}$ minimization.

Figure 16 shows this evolution compared with the SFRd required to keep the universe reionized as deduced from Madau et al. (1999). We computed this limit using a clumping factor of $C=6$ according to Pawlik et al. (2009) and consistent with 


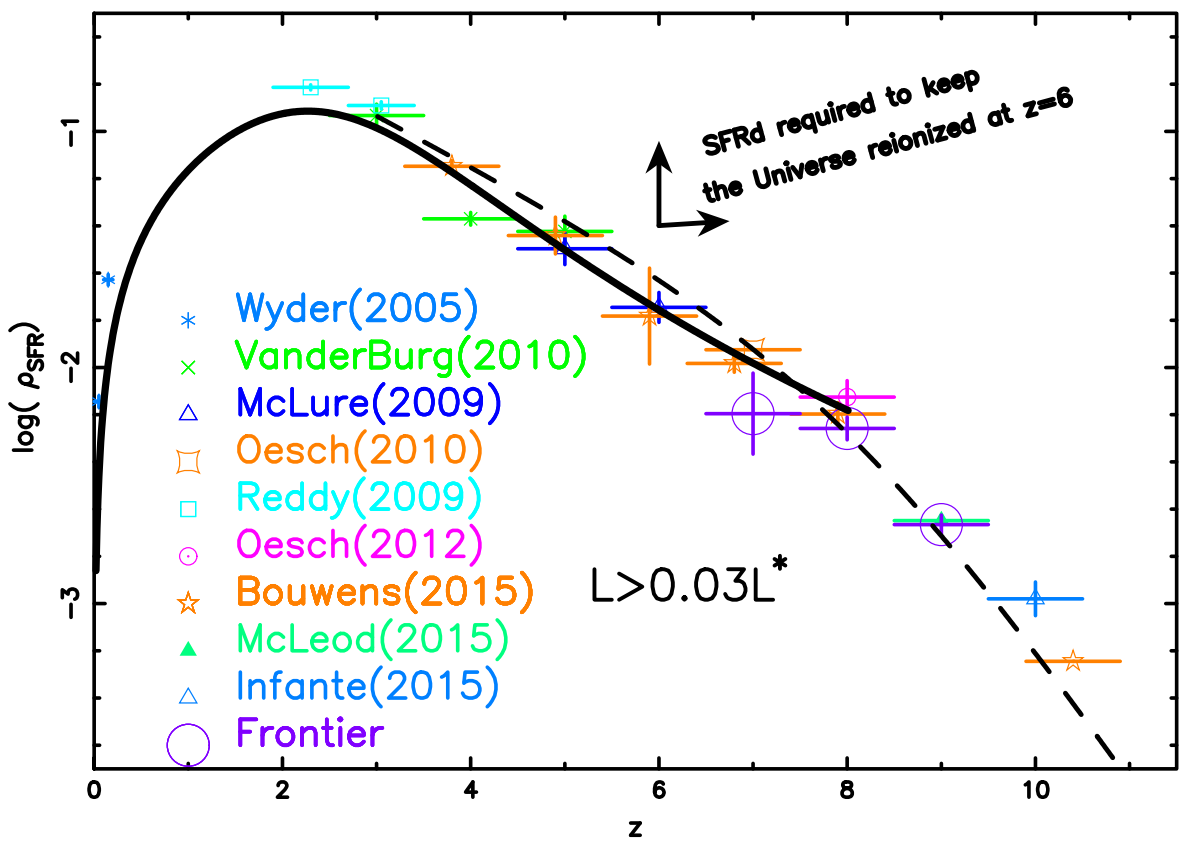

Figure 16. Evolution of the SFRd including densities deduced from the half Frontier Fields data set. We compared these results with previous measurements published in Wyder et al. (2005), van der Burg et al. (2010), McLure et al. (2009), Oesch et al. (2010b), Reddy \& Steidel (2009), Oesch et al. (2012), Bouwens et al. (2015b), and McLeod et al. (2015). Two parameterizations are overplotted: the solid line shows the shape published in Cole et al. (2001) and the dashed line displays the evolution as seen by Ishigaki et al. (2015).

recent simulations published by Kaurov \& Gnedin (2015). The escape fraction was estimated following Ferrara \& Loeb (2013) $f_{\text {esc }} \sim 0.08$, which is in good agreement with the recent upper limit published by Bouwens et al. (2015d). We corrected this value for dust extinction, which is neglected in the Madau et al. (1999) equation following the method described above. We noticed that the SFRd observed for galaxies at $z \sim 6$ with $L_{1500}>0.03 L_{z=3}^{\star}$ is still lower than what is expected to keep the universe reionized. However, if we used extreme values of the two parameters, $f_{\text {esc }} \sim 0.13$ and $C \sim 2$, we start to reconcile the observed SFRd produced by $L>0.03 L_{z=3}^{\star}$ galaxies with the SFRd required to keep the universe reionized.

\section{CONCLUSIONS}

After 1.5 years of observations, the FF program has already provided extremely deep data around four galaxy clusters, Abell 2744, MACS J0416-2403, MACS J0717+3745, and MACS 1149.5+2223, helping to increase the number of $z>6$ objects currently known. In this study, we selected $39 z>6$ objects using the Lyman break technique in the two data sets provided by this legacy program (cluster and parallel fields). We confirmed the non-detection at optical wavelength of our candidates by using an optical $\chi^{2}$ method that takes into account the position of our objects in the cluster fields. A comparison between our samples and those published using shallower optical data (e.g., CLASH) demonstrates the crucial role played by extremely deep optical data to remove extreme mid- $z$ interlopers. In this way, we have been able to identify four mid- $z$ interlopers. The size of our sample at $z \sim 6-7$ is comparable to previous findings. However, the number of $z>8$ objects is much lower than what has been found in the two first FF clusters and could be explained by CV.

We combined the $z>6$ objects selected on MACS 0717 data sets with all objects previously selected on the two first FF clusters, increasing the number of candidates to 100 . We computed photometric redshifts for our candidates from two independent approaches, $\chi^{2}$ minimization and a Bayesian method, and demonstrated that the results are in good agreement. Based on SED-fitting, we deduced the physical properties of our candidates, such as the SFR, the reddening, the stellar mass and age, and studied the relationship between several properties. Thus, we confirmed the trend observed previously in the evolution of SFR as function of galaxy mass as well as in the evolution of the size of galaxies as a function of the UV luminosity at very high redshift.

Thanks to the amplification of the light by the cluster, the majority of sources are faint and give us an opportunity to add robust constraints on the faint end of the UV LF at very high- $z$. We confirmed the shape of the UV LF at $z \sim 7$ and 8 up to $M_{1500}=-16.5$. However, due to the absence of $z>8.5$ objects behind MACS 0717 and the small number of candidates selected on the two previous FF data sets, we confirmed that the evolution of the UV LF from $z \sim 8$ to 9 could be stronger than what is observed between $z \sim 7$ and 8 . We used the LF parameterization to estimate the SFR densities produced by the galaxies up to $z \sim 10$, and confirmed the change in the evolution of SFRd between $z \sim 8$ and 10 .

All objects discussed in these papers have been selected from photometric data sets carried out with the HST. We discussed in Section 6.2 the contamination rate of our sample and demonstrated that to date it appears difficult to identify which objects could be mid- $z$ interlopers without spectroscopic observations. However, few targets identified behind MACS J0717.5+3745 are bright enough to be observed with current NIR facilities (e.g., MOSFIRE/Keck, EMIR/GTC). Spectroscopic confirmation is absolutely essential to assess the photometrically based conclusion obtained to date, particularly in light of the small number of objects currently confirmed by spectroscopy (Finkelstein et al. 2013; Oesch et al. 2015b). 
The authors thank the anonymous referee for the useful comments that strongly improved the quality of the paper. We acknowledge support from CONICYT-Chile grants BasalCATA PFB-06/2007 (NL, LI, FEB, and SK), GeminiCONICYT \#32120003 (NL), "EMBIGGEN" Anillo ACT1101 (FEB), FONDECYT 1141218 (FEB), FONDECYT Postdoctorado 3160122 (NL), 3140542 (PT) and Project IC120009 "Millennium Institute of Astrophysics (MAS)" of the Iniciativa Científica Milenio del Ministerio de Economía, Fomento y Turismo (FEB), and the French Agence Nationale de la Recherche bearing the reference ANR-09-BLAN-0234 (RP, DB). A.M. acknowledges the financial support of the Brazilian funding agency FAPESP (Postdoc fellowshipprocess number 2014/11806-9). This work was supported by award AR-13279 from the Space Telescope Science Institute (STScI), which is operated by the Association of Universities for Research in Astronomy, Inc. under NASA contract NAS 5-26555. I.C. acknowledges the support from Smithsonian Astrophysical Observatory Telescope Data Center and from the grants MD-7355.2015.2 by the research council of the president of the Russian Federation, 15-32-21062 and 15-5215050 by the Russian Foundation for Basic Research. This work is based on observations made with the NASA/ESA Hubble Space Telescope, obtained at the Space Telescope Science Institute (STScI), which is operated by the Association of Universities for Research in Astronomy, Inc., under NASA contract NAS 5-26555. The HST image mosaics were produced by the Frontier Fields Science Data Products Team at STScI. This work is based in part on observations made with the Spitzer Space Telescope, which is operated by the Jet Propulsion Laboratory, California Institute of Technology under a contract with NASA. This work utilizes gravitational lensing models produced by PIs Bradac, Ebeling, Merten \& Zitrin, Sharon, and Williams funded as part of the HST Frontier Fields program conducted by STScI. STScI is operated by the Association of Universities for Research in Astronomy, Inc. under NASA contract NAS 5-26555. The lens models were obtained from the Mikulski Archive for Space Telescopes (MAST).

\section{REFERENCES}

Atek, H., Richard, J., Jauzac, M., et al. 2015a, ApJ, in press (arXiv:1509.06764)

Atek, H., Richard, J., Kneib, J.-P., et al. 2014, ApJ, 786, 60

Atek, H., Richard, J., Kneib, J.-P., et al. 2015b, ApJ, 800, 18

Beckwith, S. V. W., Stiavelli, M., Koekemoer, A. M., et al. 2006, AJ, 132,1729

Benítez, N. 2000, ApJ, 536, 571

Bertin, E., \& Arnouts, S. 1996, A\&AS, 117, 393

Bolzonella, M., Miralles, J.-M., \& Pelló, R. 2000, A\&A, 363, 476

Bouwens, R. J., Bradley, L., Zitrin, A., et al. 2014, ApJ, 795, 126

Bouwens, R. J., Illingworth, G. D., González, V., et al. 2010, ApJ, 725, 1587

Bouwens, R. J., Illingworth, G. D., Labbe, I., et al. 2011a, Natur, 469, 504

Bouwens, R. J., Illingworth, G. D., Oesch, P. A., et al. 2011b, ApJ, 737, 90

Bouwens, R. J., Illingworth, G. D., Oesch, P. A., et al. 2012, ApJ, 754, 83

Bouwens, R. J., Illingworth, G. D., Oesch, P. A., et al. 2015a, arXiv: 1503.08228

Bouwens, R. J., Illingworth, G. D., Oesch, P. A., et al. 2015b, ApJ, 803, 34

Bouwens, R. J., Oesch, P. A., Labbe, I., et al. 2015c, ApJ, submitted (arXiv:1506.01035)

Bouwens, R. J., Smit, R., Labbe, I., et al. 2015d, ApJ, submitted (arXiv:1511.08504)

Bowler, R. A. A., Dunlop, J. S., McLure, R. J., et al. 2014, MNRAS, 440, 2810 Bradley, L. D., Trenti, M., Oesch, P. A., et al. 2012, ApJ, 760, 108

Bradley, L. D., Zitrin, A., Coe, D., et al. 2014, ApJ, 792, 76

Bruzual, G., \& Charlot, S. 2003, MNRAS, 344, 1000
Burgasser, A. J. 2007, ApJ, 658, 617

Burgasser, A. J., Burrows, A., \& Kirkpatrick, J. D. 2006a, ApJ, 639, 1095

Burgasser, A. J., Geballe, T. R., Leggett, S. K., Kirkpatrick, J. D., \& Golimowski, D. A. 2006b, ApJ, 637, 1067

Burgasser, A. J., Liu, M. C., Ireland, M. J., Cruz, K. L., \& Dupuy, T. J. 2008, ApJ, 681, 579

Burgasser, A. J., \& McElwain, M. W. 2006, AJ, 131, 1007

Burgasser, A. J., McElwain, M. W., Kirkpatrick, J. D., et al. 2004, AJ, 127, 2856

Calzetti, D., Armus, L., Bohlin, R. C., et al. 2000, ApJ, 533, 682

Chiu, K., Fan, X., Leggett, S. K., et al. 2006, AJ, 131, 2722

Coe, D., Zitrin, A., Carrasco, M., et al. 2013, ApJ, 762, 32

Cole, S., Norberg, P., Baugh, C. M., et al. 2001, MNRAS, 326, 255

Coleman, G. D., Wu, C.-C., \& Weedman, D. W. 1980, ApJS, 43, 393

Cruz, K. L., Burgasser, A. J., Reid, I. N., \& Liebert, J. 2004, ApJL, 604, L61

Cucciati, O., Tresse, L., Ilbert, O., et al. 2012, A\&A, 539, A31

Curtis-Lake, E., McLure, R. J., Dunlop, J. S., et al. 2013, MNRAS, 429, 302

Curtis-Lake, E., McLure, R. J., Dunlop, J. S., et al. 2016, MNRAS, 457, 440

Daddi, E., Dickinson, M., Morrison, G., et al. 2007, ApJ, 670, 156

Davé, R. 2008, MNRAS, 385, 147

de Barros, S., Schaerer, D., \& Stark, D. P. 2014, A\&A, 563, A81

DeMaio, T., Gonzalez, A. H., Zabludoff, A., Zaritsky, D., \& Bradač, M. 2015 MNRAS, 448, 1162

Duncan, K., \& Conselice, C. J. 2015, MNRAS, in press (arXiv:1505.01846)

Dunlop, J. S., Rogers, A. B., McLure, R. J., et al. 2013, MNRAS, 432, 3520

Ebeling, H., Barrett, E., \& Donovan, D. 2004, ApJL, 609, L49

Elbaz, D., Daddi, E., Le Borgne, D., et al. 2007, A\&A, 468, 33

Ferrara, A., \& Loeb, A. 2013, MNRAS, 431, 2826

Finkelstein, S. L., Papovich, C., Dickinson, M., et al. 2013, Natur, 502, 524

Finkelstein, S. L., Papovich, C., Salmon, B., et al. 2012, ApJ, 756, 164

Finkelstein, S. L., Ryan, R. E., Jr., Papovich, C., et al. 2015, ApJ, 810, 71

Fioc, M., \& Rocca-Volmerange, B. 1997, A\&A, 326, 950

González, V., Bouwens, R., Illingworth, G., et al. 2014, ApJ, 781, 34

Grillo, C., Suyu, S. H., Rosati, P., et al. 2015, ApJ, 800, 38

Hayes, M., Laporte, N., Pelló, R., Schaerer, D., \& Le Borgne, J.-F. 2012, MNRAS, 425, L19

Holwerda, B. W., Bouwens, R., Oesch, P., et al. 2015, ApJ, 808, 6

Hora, J. L., Carey, S., Surace, J., et al. 2008, PASP, 120, 1233

Ilbert, O., McCracken, H. J., Le Fèvre, O., et al. 2013, A\&A, 556, A55

Infante, L., Zheng, W., Laporte, N., et al. 2015, ApJ, 815, 18

Ishigaki, M., Kawamata, R., Ouchi, M., et al. 2015, ApJ, 799, 12

Johnson, T. L., Sharon, K., Bayliss, M. B., et al. 2014, ApJ, 797, 48

Jullo, E., \& Kneib, J.-P. 2009, MNRAS, 395, 1319

Jullo, E., Kneib, J.-P., Limousin, M., et al. 2007, NJPh, 9, 447

Kaurov, A. A., \& Gnedin, N. Y. 2015, ApJ, 810, 154

Kawamata, R., Ishigaki, M., Shimasaku, K., Oguri, M., \& Ouchi, M. 2015, ApJ, 804, 103

Kinney, A. L., Calzetti, D., Bohlin, R. C., et al. 1996, ApJ, 467, 38

Kirkpatrick, J. D., Looper, D. L., Burgasser, A. J., et al. 2010, ApJS, 190, 100

Kneib, J.-P., Ellis, R. S., Smail, I., Couch, W. J., \& Sharples, R. M. 1996, ApJ, 471,643

Kneib, J.-P., \& Natarajan, P. 2011, A\&ARv, 19, 47

Krist, J. E., Hook, R. N., \& Stoehr, F. 2011, Proc. SPIE, 8127, 81270J

Labbé, I., González, V., Bouwens, R. J., et al. 2010, ApJL, 716, L103

Labbé, I., Oesch, P. A., Bouwens, R. J., et al. 2013, ApJL, 777, L19

Laporte, N., Streblyanska, A., Clement, B., et al. 2014, A\&A, 562, L8

Laporte, N., Streblyanska, A., Kim, S., et al. 2015, A\&A, 575, A92

Liebert, J., \& Burgasser, A. J. 2007, ApJ, 655, 522

Looper, D. L., Kirkpatrick, J. D., \& Burgasser, A. J. 2007, AJ, 134, 1162

Lorenzoni, S., Bunker, A. J., Wilkins, S. M., et al. 2011, MNRAS, 414, 1455

Lotz, J., Mountain, M., Grogin, N. A., et al. 2014, BAAS, 223, 254.01

Madau, P. 1995, ApJ, 441, 18

Madau, P., Haardt, F., \& Rees, M. J. 1999, ApJ, 514, 648

Maizy, A., Richard, J., de Leo, M. A., Pelló, R., \& Kneib, J. P. 2010, A\&A, 509, A105

Maraston, C., Pforr, J., Renzini, A., et al. 2010, MNRAS, 407, 830

McElwain, M. W., \& Burgasser, A. J. 2006, AJ, 132, 2074

McGreer, I. D., Mesinger, A., \& D'Odorico, V. 2015, MNRAS, 447, 499

McLean, I. S., Steidel, C. C., Epps, H. W., et al. 2012, Proc. SPIE, 8446, $84460 \mathrm{~J}$

McLeod, D. J., McLure, R. J., Dunlop, J. S., et al. 2015, MNRAS, 450, 3032

McLure, R. J., Cirasuolo, M., Dunlop, J. S., Foucaud, S., \& Almaini, O. 2009, MNRAS, 395, 2196

McLure, R. J., Dunlop, J. S., Bowler, R. A. A., et al. 2013, MNRAS, 432, 2696

McLure, R. J., Dunlop, J. S., de Ravel, L., et al. 2011, MNRAS, 418, 2074

Medezinski, E., Umetsu, K., Nonino, M., et al. 2013, ApJ, 777, 43 
Merten, J., Coe, D., Dupke, R., et al. 2011, MNRAS, 417, 333

Molino, A., Benítez, N., Moles, M., et al. 2014, MNRAS, 441, 2891

Moustakas, J., Coil, A. L., Aird, J., et al. 2013, ApJ, 767, 50

Oesch, P. A., Bouwens, R. J., Carollo, C. M., et al. 2010a, ApJL, 709, L21

Oesch, P. A., Bouwens, R. J., Carollo, C. M., et al. 2010b, ApJL, 725, L150

Oesch, P. A., Bouwens, R. J., Illingworth, G. D., et al. 2010c, ApJL, 709, L16

Oesch, P. A., Bouwens, R. J., Illingworth, G. D., et al. 2012, ApJ, 759, 135

Oesch, P. A., Bouwens, R. J., Illingworth, G. D., et al. 2013, ApJ, 773, 75

Oesch, P. A., Bouwens, R. J., Illingworth, G. D., et al. 2014, ApJ, 786, 108

Oesch, P. A., Bouwens, R. J., Illingworth, G. D., et al. 2015a, ApJ, 808, 104

Oesch, P. A., van Dokkum, P. G., Illingworth, G. D., et al. 2015b, ApJL, 804, L30

Oke, J. B., \& Gunn, J. E. 1983, ApJ, 266, 713

Ono, Y., Ouchi, M., Curtis-Lake, E., et al. 2013, ApJ, 777, 155

Pawlik, A. H., Schaye, J., \& van Scherpenzeel, E. 2009, MNRAS, 394, 1812

Peng, C. Y., Ho, L. C., Impey, C. D., \& Rix, H.-W. 2010, AJ, 139, 2097

Polletta, M., Tajer, M., Maraschi, L., et al. 2007, ApJ, 663, 81

Postman, M., Coe, D., Benítez, N., et al. 2012, ApJS, 199, 25

Puget, P., Stadler, E., Doyon, R., et al. 2004, Proc. SPIE, 5492, 978

Reddy, N. A., \& Steidel, C. C. 2009, ApJ, 692, 778

Reid, I. N., Lewitus, E., Burgasser, A. J., \& Cruz, K. L. 2006, ApJ, 639, 1114

Richard, J., Jauzac, M., Limousin, M., et al. 2014, MNRAS, 444, 268

Roberts-Borsani, G. W., Bouwens, R. J., Oesch, P. A., et al. 2015, ApJ, submitted (arXiv:1506.00854)

Schaerer, D., \& de Barros, S. 2010, A\&A, 515, A73

Schaerer, D., de Barros, S., \& Sklias, P. 2013, A\&A, 549, A4

Schechter, P. 1976, ApJ, 203, 297

Schenker, M. A., Robertson, B. E., Ellis, R. S., et al. 2013, ApJ, 768, 196

Schiminovich, D., Ilbert, O., Arnouts, S., et al. 2005, ApJL, 619, L47

Schmidt, K. B., Treu, T., Bradač, M., et al. 2016, ApJ, 818, 38

Schmidt, K. B., Treu, T., Brammer, G. B., et al. 2014, ApJL, 782, L36
Schroeder, J., Mesinger, A., \& Haiman, Z. 2013, MNRAS, 428, 3058

Sheppard, S. S., \& Cushing, M. C. 2009, AJ, 137, 304

Siegler, N., Close, L. M., Burgasser, A. J., et al. 2007, AJ, 133, 2320

Silva, L., Granato, G. L., Bressan, A., \& Danese, L. 1998, ApJ, 509, 103

Smit, R., Bouwens, R. J., Franx, M., et al. 2015, ApJ, 801, 122

Stark, D. P., Schenker, M. A., Ellis, R., et al. 2013, ApJ, 763, 129

Steidel, C. C., Adelberger, K. L., Giavalisco, M., Dickinson, M., \& Pettini, M. 1999, ApJ, 519, 1

Tasca, L. A. M., Le Fèvre, O., Hathi, N. P., et al. 2015, A\&A, 581, A54

Taylor, M. B. 2005, in ASP Conf. Ser. 347, Astronomical Data Analysis Software and Systems XIV, ed. P. Shopbell, M. Britton, \& R. Ebert (San Francisco, CA: ASP), 29

Trenti, M., Bradley, L. D., Stiavelli, M., et al. 2011, ApJL, 727, L39

Trenti, M., \& Stiavelli, M. 2008, ApJ, 676, 767

van der Burg, R. F. J., Hildebrandt, H., \& Erben, T. 2010, A\&A, 523, A74

Vanzella, E., Fontana, A., Zitrin, A., et al. 2014, ApJL, 783, L12

Vernet, J., Dekker, H., D’Odorico, S., et al. 2011, A\&A, 536, A105

Wilkins, S. M., Bunker, A. J., Stanway, E., Lorenzoni, S., \& Caruana, J. 2011, MNRAS, 417, 717

Windhorst, R. A., Cohen, S. H., Hathi, N. P., et al. 2011, ApJS, 193, 27

Wright, E. L. 2006, PASP, 118, 1711

Wyder, T. K., Treyer, M. A., Milliard, B., et al. 2005, ApJL, 619, L15

Yan, H., Finkelstein, S. L., Huang, K.-H., et al. 2012, ApJ, 761, 177

Zaroubi, S. 2013, in The First Galaxies, Astrophysics and Space Science Library, Vol. 396, ed. T. Wiklind, B. Mobasher, \& V. Bromm (Berlin: Springer), 45

Zheng, W., Postman, M., Zitrin, A., et al. 2012, Natur, 489, 406

Zheng, W., Shu, X., Moustakas, J., et al. 2014, ApJ, 795, 93

Zitrin, A., Fabris, A., Merten, J., et al. 2015a, ApJ, 801, 44

Zitrin, A., Labbe, I., Belli, S., et al. 2015b, ApJL, 810, L12

Zitrin, A., Zheng, W., Broadhurst, T., et al. 2014, ApJL, 793, L12 